\title{
Investigation of Rotor-Airframe Interaction Noise Associated with Small-Scale Rotary-Wing Unmanned Aircraft Systems
}

\author{
Nikolas S. Zawodny \\ Research Aerospace Engineer \\ D. Douglas Boyd Jr. \\ Aeroacoustics Branch, NASA Langley Research Center \\ Hampton, VA 23681-2199
}

\begin{abstract}
In this study, hover acoustic measurements are taken on isolated rotor-airframe configurations representative of smallscale, rotary-wing unmanned aircraft systems (UAS). Each rotor-airframe configuration consists of two fixed-pitch blades powered by a brushless motor, with a simplified airframe geometry intended to represent a generic multicopter arm. In addition to acoustic measurements, CFD-based aeroacoustic predictions are implemented on a subset of the experimentally tested rotor-airframe configurations in an effort to better understand the noise content of the rotor-airframe systems. Favorable agreements are obtained between acoustic measurements and predictions, based on both time- and frequency-domain post-processing techniques. Results indicate that close proximity of airframe surfaces result in the generation of considerable tonal acoustic content in the form of harmonics of the rotor blade passage frequency (BPF). Analysis of the acoustic prediction data shows that the presence of the airframe surfaces can generate noise levels either comparable to or greater than the rotor blade surfaces under certain rotor tip clearance conditions. Analysis of the on-surface Ffowcs Williams and Hawkings (FW-H) source terms provide insight as to the predicted physical noise-generating mechanisms on the rotor and airframe surfaces.
\end{abstract}

\section{NOMENCLATURE}

c Rotor blade element chord, mm

$L \quad$ Overall Sound Pressure Level (OASPL), dB

$N_{\text {revs }} \quad$ Number of rotor revolutions

$R \quad$ Rotor tip radius, $\mathrm{mm}$

$T \quad$ Rotor thrust, N

$W \quad$ Width of airframe cross-section, $\mathrm{mm}$

$\Delta \quad$ Rotor-airframe tip clearance, $\mathrm{mm}$

$\varepsilon_{r} \quad$ Normalized autospectral random uncertainty, $\%$

$\theta \quad$ Observer elevation angle, deg.

$\Sigma \quad$ Acoustic surface of constant observer time

$\sigma_{p_{\text {rms }}} \quad$ Standard deviation of pressure, $\mathrm{Pa}$

$\phi \quad$ Observer azimuthal angle, deg.

$\Omega \quad$ Rotor rotation rate, RPM

\section{Subscripts}

A A-weighting

$T \quad$ Tonal contribution

\section{INTRODUCTION}

Since May of 2014, private companies have been able to file petitions for exemption from obtaining airworthiness certificates to permit commercial unmanned aircraft system (UAS) operations (Ref. 1). Such exemptions are permitted under Section 333 of the Modernization and Reform Act of Presented at the AHS 73rd Annual Forum, Fort Worth, Texas, May 9-11, 2017.
2012 implemented by the Federal Aviation Administration (FAA) (Ref. 2). Of the first 1,000 commercial UAS exemptions granted by the FAA, 90\% are rotary-wing in nature, 99\% of which are multi-copters (Ref. 3). The increased presence of such UAS in the national air space (NAS) of the United States over the past several years has led to a need for a better understanding of the possible impacts these vehicles will have on the civilian population. One of the impacts likely to affect the usage of these vehicles is how they are acoustically perceived by humans.

The ability to predict the noise of UAS can be important to the selection and/or design process of vehicles to meet specified mission criteria. Previous work has shown that both high-fidelity physics-based simulations and low-fidelity semi-analytical prediction methods yield favorable acoustic comparisons with experimental measurements for cases of small isolated rotors in clean hover conditions (Ref. 4). These prediction methods included acoustic tonal predictions using a Ffowcs Williams and Hawkings (FW-H) acoustic solver (Ref. 5) based on rotor surface pressures computed from CFD (OVERFLOW2) (Ref. 6) or blade element analysis using the Propeller Analysis System of the Aircraft NOise Prediction Program (ANOPP-PAS) (Ref. 7), as well as semianalytical broadband acoustic predictions based on the incorporation of an airfoil self-noise model (Ref. 8). However, the incorporation of an airframe surface below the rotor disk can greatly complicate the resulting flow field. The presence of an airframe, such as a wing under a hovering tiltrotor, is reminiscent of a multi-copter arm located below the rotor disk plane. In the case of the tiltrotor, the presence of the wing under the hovering rotor both obstructs the downwash causing the flow to turn spanwise along the wing, and acts as a partial 
ground plane causing a decrease in the inflow velocity seen by the rotor as it passes over the wing (Ref. 9). The authors of Refs. 9 and 10 postulated that the resulting primary noise generation mechanism, labeled the "fountain effect," was associated with the localized changes in blade loading as the blade passes through the "fountain" area affected by the downwash recirculation. However, this was primarily for the purpose of modeling the rotor flow field, and does not account for the possibility that the wing surface itself could play an important role in the resulting noise generation. Similarly, the airframe surfaces of a UAS may need to be accounted for in the noise prediction process.

\section{TECHNICAL APPROACH}

\section{Experimental Setup}

Experiments were performed in the Structural Acoustic Loads and Transmission (SALT) anechoic chamber facility at the NASA Langley Research Center (Refs. 4,11). A total of five $6.35 \mathrm{~mm}$ Type 4939 free-field Brüel \& Kjaer microphones in an arc array configuration are positioned in the acoustic farfield of the rotor test stand. The microphones are positioned at a radial distance of $1.905 \mathrm{~m}$ from the rotor hub, which corresponds to approximately $16 R$ for the rotor tested in this study. The microphone array is divided into elevation and azimuthal sub-arrays, the angular locations of which are defined in Table 1 . The rotor test stand itself was constructed so that the plane of the rotor would stand $2.29 \mathrm{~m}$ above the floor wedge tips, which corresponds to half of the room height. Pictures of the test setup are shown in Figure 1.

Table 1. Experimental nominal microphone locations

\begin{tabular}{lr}
\hline \hline Microphone & $(\theta, \phi)^{a}$ \\
\hline M1 & $(0.0,0.0)$ \\
M2 & $(-22.5,0.0)$ \\
M3 & $(-45.0,0.0)$ \\
M4 & $(-45.0,+45.0)$ \\
M5 & $(-45.0,+90.0)$ \\
\hline \hline
\end{tabular}

${ }^{a}$ Angular units in degrees; all microphones located at a radial location of $r=16 R$ from rotor hub.

The Rotor and Rotor Stand The rotor tested in this study is a carbon fiber rotor similar in geometry to that of a rotor on a commercially available UAS. This rotor is herein referred to as the DJI-CF rotor. The tip radius of this rotor is $R=119.2$ $\mathrm{mm}$. Geometric details of this rotor as well as a detailed description of the rotor test stand may be found in Ref. 4. One addition to the rotor test stand is a neoprene damping material, which was positioned directly underneath the load cell for the purpose of reducing noise due to motor and test stand vibrations.

Airframe Support Stand The airframe support stand was designed to hold a simple airframe component such that it would be physically separate from the rotor stand. The support stand itself was positioned approximately $4 R$ away from

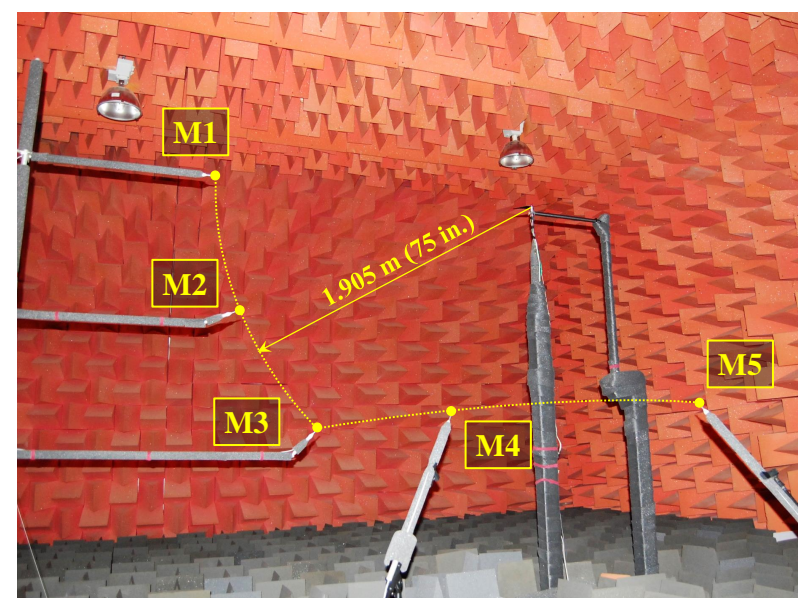

(a) Test Setup in SALT Facility

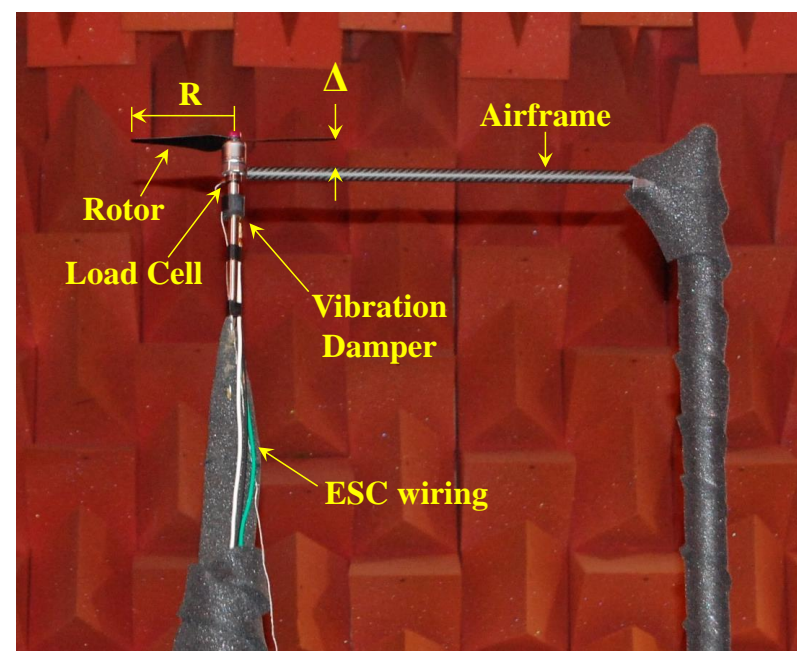

(b) Rotor and Airframe Support Stands

Fig. 1. Components and geometric parameters of experimental test setup.

the rotor hub, and supported airframe geometries in a cantilever fashion, aligned perpendicular to the rotor shaft. A total of four different airframe geometries were tested in this study. The first three of these are constant cross-section hollow carbon fiber circular tubes, each with a different crosssectional dimension (see Fig. 2). These simple geometries were selected for investigation due to their common usage on numerous generic multi-copter platforms. The fourth airframe geometry is a conical section whose dimensions are similar to a readily available commercial off-the-shelf (COTS) vehicle. This geometry was tested to provide insight into differences in noise generation due to airframes of varying cross-section, specifically when the airframe cross-section becomes considerably larger than the reference cross-section of the rotor. For this study, this reference cross-sectional length is the rotor chord at $75 \%$ tip radius, or $c_{0.75 R}=14.8 \mathrm{~mm}$. The airframe components were positioned at various vertical tip clearance locations, $\Delta$, which were measured relative to the approximate 1/4-chord location of the rotor tip (see Fig. 1(b)). Geometric details of the conical airframe are provided in Figure 3. 


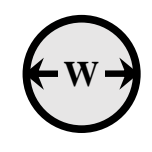

(a)

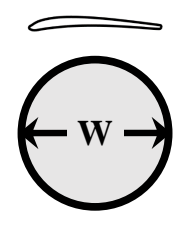

(b)

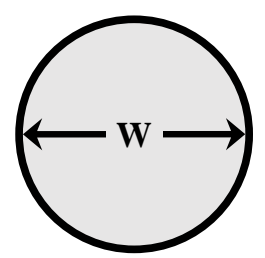

(c)

\begin{tabular}{crc}
\hline \hline Figure & $W(\mathrm{~mm})$ & $W / c_{0.75 R}$ \\
\hline $2(\mathrm{a})$ & 12.0 & 0.81 \\
$2(\mathrm{~b})$ & 16.0 & 1.08 \\
2(c) & 25.0 & 1.69 \\
\hline \hline
\end{tabular}

Fig. 2. Images and dimensions of tested constant crosssection (generic) airframes. Note: Rotor $75 \%$ spanwise section superimposed to scale for reference.

Data Acquisition In addition to the microphones mentioned previously, unsteady data were acquired on a single-axis load cell for measuring rotor thrust located directly below the motor (see Fig. 1(b)), a laser sensor tachometer for measuring the mechanical rotation rate of the rotor-motor assembly, and a probe for measuring the alternating current (AC) signal between one of the three phases between the electronic speed controller (ESC) and the motor. The ESC-motor AC signal was acquired in order to better distinguish between rotor- and motor-generated noise. While data were acquired for a duration of 20 seconds at a sampling rate of $80 \mathrm{kHz}$, only ten seconds of data were used for calculation of acoustic spectra. This time range was selected to be within the first 15 seconds of data acquisition in order to ensure that the rotorgenerated noise was not contaminated by flow recirculation effects. All unsteady data were acquired using 24-bit National Instruments PXI-4472B modules installed in a PXI1045 chassis.

Testing Conditions The experimental testing conditions are summarized in Table 2. As the table shows, two rotor rotation rate conditions were analyzed, with the goals of investigating the effects of different geometric parameters. The primary rotation rate of $\Omega=5400$ RPM was measured for the purpose of ascertaining the effects of different airframe proximities to the rotor plane $(\Delta / R)$. This rotation rate was selected because it represents a typical hover condition for a UAS this size.

Table 2. Experimental testing conditions.

\begin{tabular}{llll}
\hline \hline Case & $\Omega(\mathrm{RPM})$ & $W / c_{0.75 R}$ & $\Delta / R$ \\
\hline Isolated Rotor & 5400,6000 & $\mathrm{~N} / \mathrm{A}$ & $\mathrm{N} / \mathrm{A}$ \\
Generic Airframe & 6000 & 0.81 & -0.3 \\
& 5400,6000 & 1.08 & $-0.1 \Rightarrow-0.5^{a}$ \\
& 6000 & 1.69 & -0.3 \\
Conical Airframe & 5400 & $3.45^{b}$ & $-0.1 \Rightarrow-0.5^{b c}$ \\
\hline \hline
\end{tabular}

${ }^{a}$ Varied in increments of $\Delta / R=0.1$.

${ }^{b}$ As measured at plane of rotor tip (See Fig. 3(b)).

${ }^{c}$ Varied in increments of $\Delta / R=0.2$.

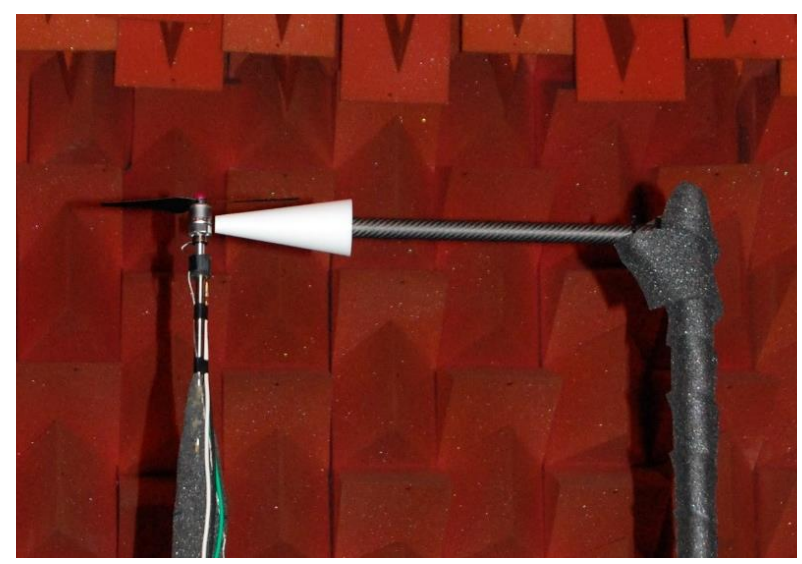

(a) Conical airframe in experimental setup

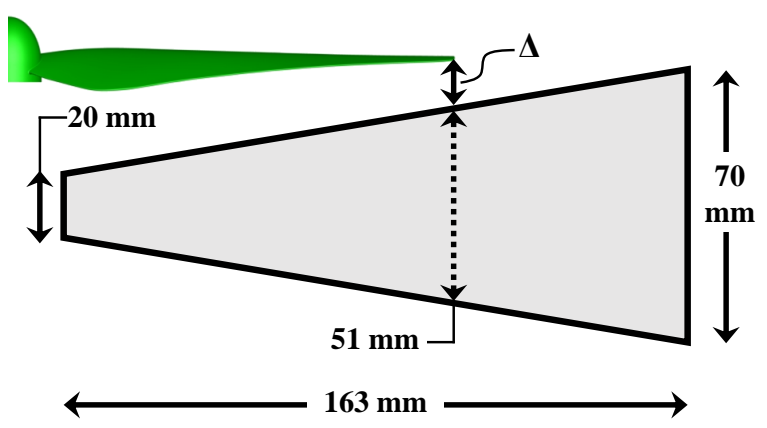

(b) Geometry of conical airframe

Fig. 3. Image and geometric details of tested conical airframe. (Note: Image (b) drawn to scale for case of $\Delta / R=$ -0.1.)

The generic airframe shown in Fig. 2(b) was selected for a fine resolution variation of airframe proximity because it has a cross-sectional width that is comparable to the rotor reference chord dimension, $W / c_{0.75 R}=1.08$. The conical airframe proximity to the rotor plane was also varied at coarser resolution for reference comparison with the generic airframe. The effects of different airframe cross-sections were then examined at a slightly higher rotation rate of $\Omega=6000$ RPM for a common tip clearance of $\Delta / R=-0.3$, where the negative tip clearance numbers indicate that the airframe is below the rotor. This rotation rate was selected in order to increase the system signal-to-noise ratio (SNR) for an airframe proximity condition expected to yield lower overall noise levels. The tip clearances for these tests were chosen because they represent typical rotor-airframe spacings of generic multi-copter vehicles. Finally, isolated rotor data were taken at both rotation rates for reference.

\section{Noise Prediction Method}

Deterministic noise predictions were carried out utilizing unsteady surface pressures computed using CFD, which were then used as impermeable loading and geometry data into a Ffowcs Williams and Hawkings (FW-H) solver. CFD simulations were performed using OVERFLOW2, an un- 
steady Reynolds-averaged Navier-Stokes (URANS) code, while noise predictions were carried out using the PSUWOPWOP code. The overall noise prediction process herein referred to as OF2-PSW - is similar to that previously reported in Ref. 4, but with some modifications to the CFD implementation.

The computational grid was constructed using overset grid generation via Chimera Grid Tools (Ref. 12). The "nearbody" grids are structured, body-fitted grids around the solid surfaces (rotor blades, hub, airframe). These near-body grids are initially encased in a large, user-defined Cartesian grid region (Level-1 grid), with a uniform grid spacing of $20 \%$ of $c_{0.75 R}$ in all directions. OVERFLOW2 automatically generates grids between the Level-1 grid and the far field. In the Cartesian Levell off-body grids, adaptive mesh refinement (AMR) is used. The off-body grids were permitted to refine up to two levels finer than their starting level based on an undivided squared difference criterion of the primary flow variables (density, momentum, and stagnation energy density). In other words, the initial first level Cartesian off-body grid was set to have a grid spacing corresponding to $20 \%$ of $c_{0.75 R}$, and allowed to refine down to a spacing of $5 \%$ of $c_{0.75 R}$. A total of 19 rotor revolutions were simulated for each case, the final one of which was used for the noise predictions. The first ten revolutions were simulated at a physical time resolution corresponding to $2.5^{\circ}$ azimuthal increments, after which the time resolution was refined to $0.25^{\circ}$ azimuthal increments. The surface pressure data were input into the acoustic solver in $1^{\circ}$ azimuthal increments and run as a periodic case.

A total of six CFD cases were run, which are summarized in Table 3. Note that all CFD runs were done for a rotor rotation rate of $\Omega=5400 \mathrm{RPM}(90 \mathrm{~Hz})$. For visualization, a crosssection view of the core CFD computational domain taken from the last time step of the final rotor revolution for the conical airframe case is shown in Figure 4. More specifically, this figure shows the grids that make up approximately $98 \%$ of the total 81 million grid points that define the domain (at this time step). Note that the remaining six off-body grid levels, which account for the final $2 \%$ of the computational domain, are not shown in Figure 4. A considerable computation time benefit was observed with the implementation of AMR for the off-body grid generation, without sacrificing the quality of the simulation results. More specifically, the simulation procedure mentioned previously for the conical airframe case took approximately 23 hours to simulate using 720 cores on the NAS Pleiades supercomputer. For an identical simulation procedure not utilizing AMR, this would be increased to 132 hours for a computational domain consisting of 161 million grid points.

Table 3. OVERFLOW2 simulation cases.

\begin{tabular}{llr}
\hline \hline Case & $W / c_{0.75 R}$ & Tip Clearance $(\Delta / R)$ \\
\hline Isolated Rotor & N/A & N/A \\
Generic Airframe & 1.08 & $+0.1,-0.1,-0.3,-0.5$ \\
Conical Airframe & $3.45^{a}$ & $-0.1^{a}$ \\
\hline \hline
\end{tabular}

${ }^{a}$ As measured at plane of rotor tip (See Fig. 3(b)).

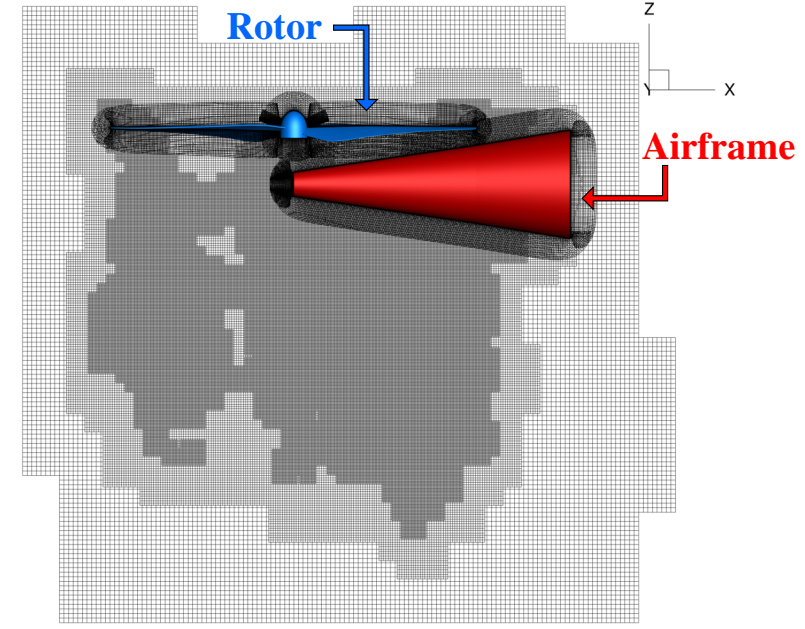

Fig. 4. Cross-section view of near-body and first three AMR off-body grid levels of OVERFLOW2 computational domain for case of rotor-conical airframe interaction.

\section{Measured Acoustic Data Post-Processing}

Acoustic data were processed using three methods, which are described in detail in Ref. 4. They are summarized here however, as follows:

1. Random data set: narrowband spectra computed using fast Fourier Transform (FFT)

2. Un-filtered rotor revolution: Mean rotor revolution time history computed, subtracted from time record to retain random noise components

3. BPF harmonic-filtered data set: Acoustic time series filtered to retain only harmonics of BPF

Method 1 provides an overall view of the spectral content of the motor-rotor-airframe system, without distinguishing between the periodic and random components of noise. Acoustic spectra using method 1 were computed using a Hanning window with $75 \%$ overlap and a frequency resolution of 5 Hz. For ten seconds of data, this yields a total of 50 blocks with 16,000 samples per block. This results in an autospectral random uncertainty of $\varepsilon_{r}=9.90 \%$, and a random SPL uncertainty of $u_{r, \mathrm{SPL}} \in\{-0.45,+0.41\} \mathrm{dB}$ (Ref. 13). Methods 2 and 3 are similar in nature to each other, extracting the periodic noise components of the system. More specifically, method 2 allows for the separation of periodic and random noise components in the time domain. Narrowband spectra are then computed on the mean revolution time history and residual random time series for a common frequency resolution of $5 \mathrm{~Hz}$. Processing the mean and residual time series with a common frequency resolution required replicating the mean revolution time history by the number of revolution time blocks. Finally, the purpose of method 3 is to more accurately compare acoustic amplitudes between predictions and experiment in the time domain. This method is applied in a nearly identical manner to both experimental and computational data 
sets, the only difference being in the requirement of replicating the single revolution of prediction data to remove the transient effects of the filtering process. Acoustic amplitudes of each BPF harmonic are computed by calculating the RMS of the ensemble-averaged pressure time history across all rotor revolutions according to

$$
\mathrm{SPL}_{n * B P F}=20 \log _{10}\left(\frac{\bar{p}_{\mathrm{rms}}}{p_{\text {ref }}}\right) .
$$

The uncertainties of these tonal amplitudes are approximated by

$$
u_{\mathrm{SPL}_{n * B P F}}=20 \log _{10}\left(\frac{\bar{p}_{\mathrm{rms}} \pm 2.0 \sigma_{p_{\mathrm{rms}}} / \sqrt{N_{\mathrm{revs}}}}{p_{\mathrm{ref}}}\right)
$$

The spectral uncertainties associated with method 3 will be presented in the form of error bars for the remainder of this paper.

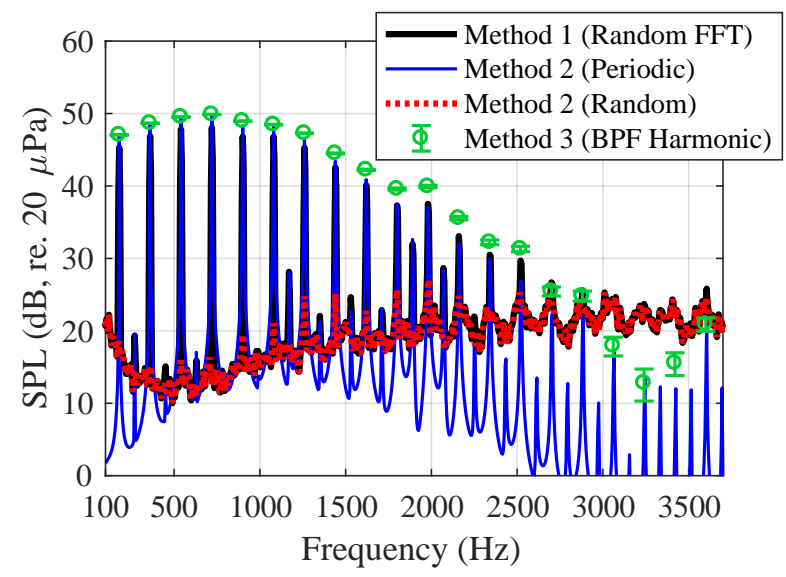

(a) Processing methods in frequency domain

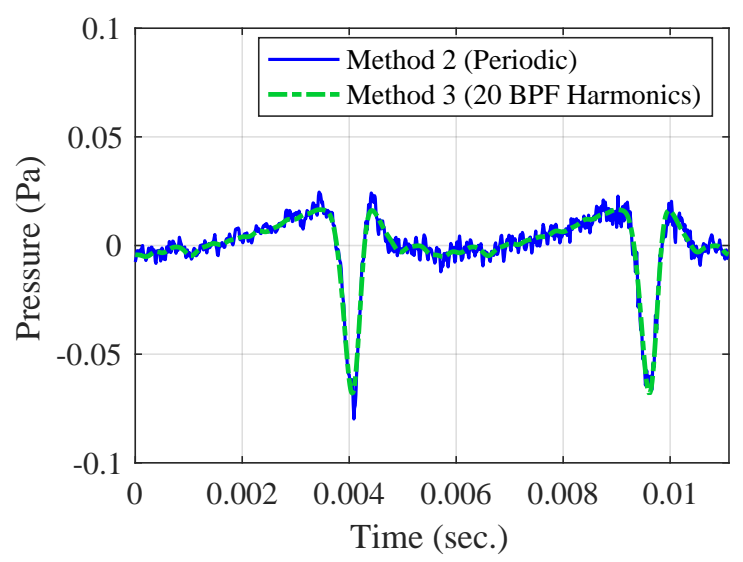

(b) Mean rotor revolution acoustic time histories

Fig. 5. Visualization of experimental acoustic postprocessing techniques applied to a rotor-airframe interaction case of high tonal content. (Case: $\Omega=5400$ RPM, $W / c_{0.75 R}=\mathbf{1 . 0 8}, \Delta / R=\mathbf{- 0 . 1}$, microphone M5)

Illustrations of these processing techniques are provided in Figure 5 for a rotor-airframe interaction case of high tonal content, applied to microphone M5. As the frequency domain results of Figure 5(a) show, method 2 effectively parses the original random FFT signal (method 1) into periodic and random components. Furthermore, the time series representations of methods 2 and 3 in Figure 5(b) show the common periodic revolution behavior of the rotor, with method 3 providing an idealized representation of the rotor revolution based solely on the first 20 harmonics of the BPF. Again, the purpose of method 3 is to provide a common basis with which to compare acoustic prediction results from OF2-PSW.

Overall sound pressure levels (OASPLs) are also computed for the periodic (tonal, $T$ ) noise spectra computed from method 2. This includes both unweighted and A-weighted OASPL metrics (Ref. 14). This is done to quantify the relative roles of the tonal and broadband content of representative rotor-airframe interactions using common sound metrics. A visualization of the effect of A-weighting on a representative rotor-airframe interaction case is presented in Figure 6. As this figure shows, application of A-weighting to the spectrum considerably reduces the amplitude of the first three BPF harmonics, as well as the broadband content for frequencies above $7 \mathrm{kHz}$. The effects of this on the integrated levels are documented in the following sections. Note that for this study, primary emphasis is placed on the periodic components of noise. Therefore, a conservative frequency range is defined with the intent of capturing the aerodynamically-generated effects of the different rotor-airframe configurations on the radiated noise, and not being misled by the tonal content due to motor noise and test stand vibrations. For purposes that will be clarified in the Results section, all experimental OASPLs are computed across a common frequency range of $100 \mathrm{~Hz}$ $\leq f \leq 20 * \mathrm{BPF}$. This frequency range is found to appropriately capture the harmonic content associated with the rotorairframe aerodynamics.

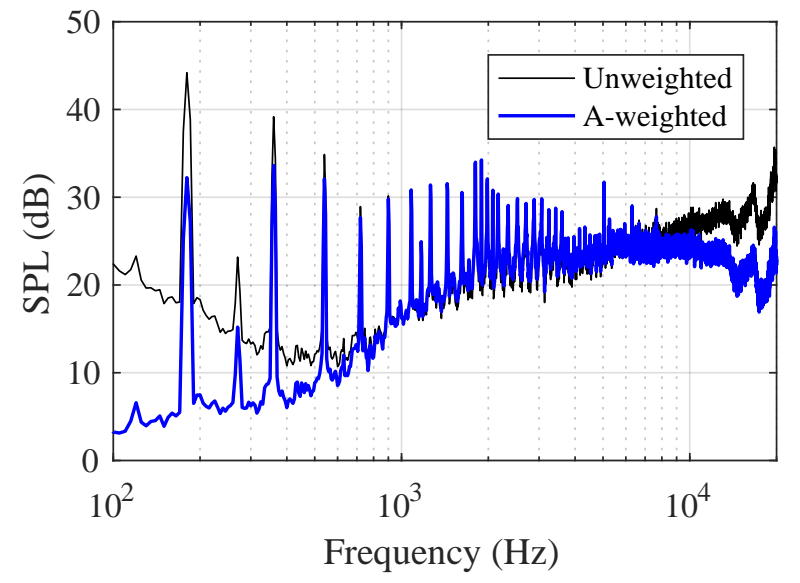

Fig. 6. Unweighted and A-weighted acoustic narrowband spectra (M5). (Case: $\Omega=5400$ RPM, BPF $=180 \mathrm{~Hz}$, $\left.W / c_{0.75 R}=\mathbf{1 . 0 8}, \Delta / R=\mathbf{- 0 . 3}\right)$

\section{RESULTS}

The results of this study are divided into two sections. The first section presents spectral comparisons between the different rotor-airframe configurations experimentally measured, 
with interpretations of rotor-generated tonal and broadband noise contributions along with supporting evidence of motor and test stand noise contributions. Effects of airframe spacing and airframe size on the radiated acoustics are investigated and discussed. The second section presents results using the deterministic extraction post-processing techniques discussed previously, with comparisons between experiments and predictions. The aeroacoustic prediction data are then utilized to identify relative noise contributions of rotor and airframe surfaces, as well as to assist with identification of the physical noise-generating mechanisms for two simulation cases containing prominent rotor-airframe interaction noise.

\section{Experimental Spectral Observations}

Effects of Airframe Proximity The effects of airframe proximity were investigated primarily for the generic airframe configuration of $W / c_{0.75 R}=1.08$. A summary of randomlyprocessed acoustic spectra for three different airframe proximity conditions are provided in Figure 7, along with isolated rotor and unloaded motor only data. Microphones M1, M3, and M5 were chosen to illustrate these spectra because they represent the elevation and azimuthal extents of the microphone sub-arrays. It is worth noting that microphones M3 and M5 show consistent spectral content for the isolated rotor case, which demonstrates the reliable acoustic quality of the experimental apparatus. If attention is focused on M5, it can be seen that the case of $\Delta / R=-0.1$ produces a harmonicrich spectrum with the second through seventh BPF harmonics having an acoustic amplitude greater than or equal to the BPF acoustic amplitude itself. Microphone M3 shows a similar behavior, but with harmonic amplitudes all being at least $5 \mathrm{~dB}$ below the BPF amplitude. Increasing the distance between the rotor and airframe to $\Delta / R=-0.3$ shows a considerable decrease in harmonic content, and a further increase to $\Delta / R=-0.5$ reveals acoustic spectra very similar to the case of an isolated rotor. This trend is seen in all microphones. The unloaded motor only spectra illustrate discrete frequencies of noise contamination across frequency ranges of $990 \mathrm{~Hz} \leq f \leq$ $1.26 \mathrm{kHz}$ and $4 \leq f \leq 6.5 \mathrm{kHz}$. The former of these frequency ranges is related to the cogging torque generated due to the rotation of the motor housing, while the latter is related to the switching frequency of the electronic speed controller. It is this high-frequency (and in certain cases, high-amplitude) noise not due to rotor-airframe aerodynamics, that warrants a more restrictive frequency range for tonal OASPL calculations.

It is interesting to note how the tonal amplitudes are larger for M5 than for M3, and how M5 is oriented normal to the centerplane of the rod while M3 is coplanar with the rod. This is an indication that the rod may be a prominent, directive noise contributor for this case. Furthermore, it is interesting to note that few changes are observed in the broadband spectral content regardless of the airframe configuration. As was found in Ref. 4, this broadband content for the case of an isolated rotor has been characterized to be due primarily to turbulent boundary layer-trailing edge (TBL-TE) and trailing
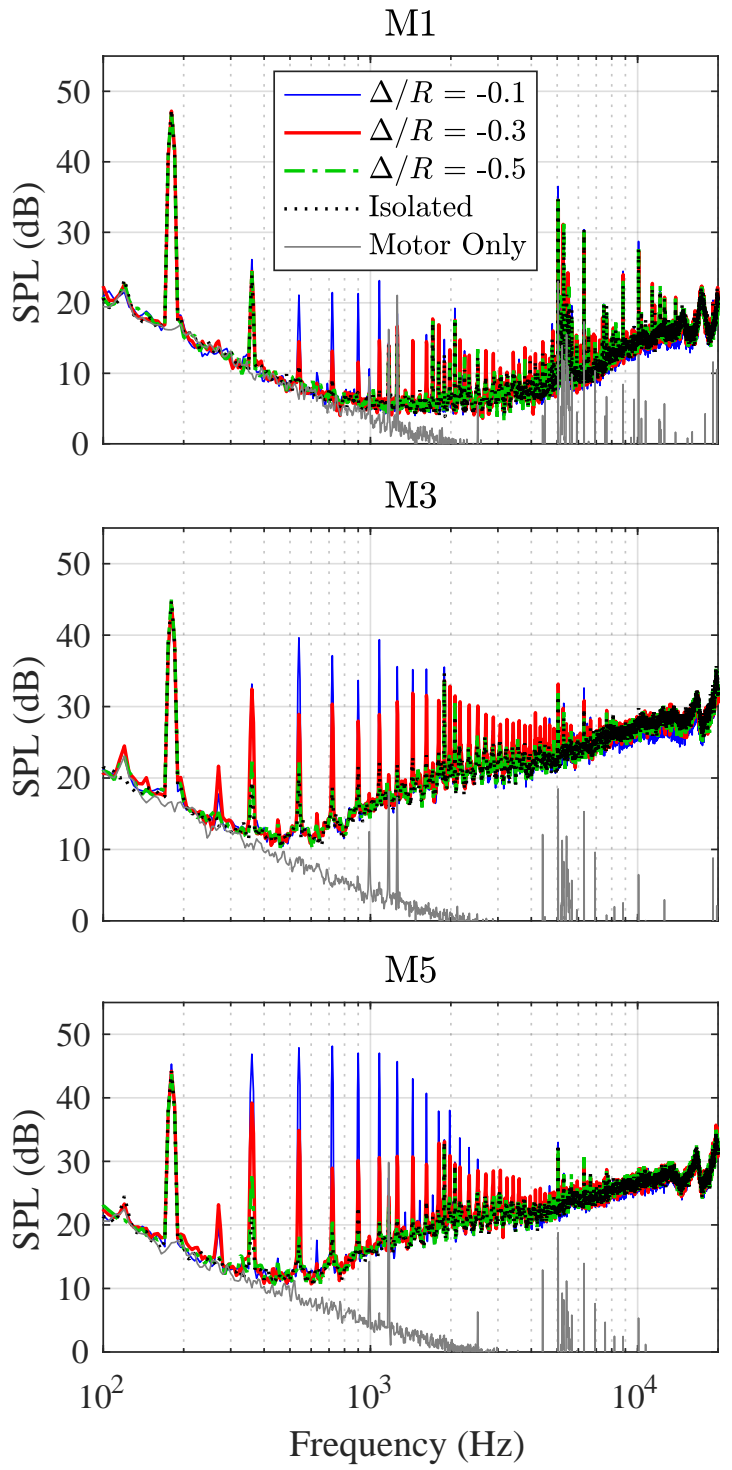

Fig. 7. Acoustic narrowband spectra at a frequency resolution of $5 \mathrm{~Hz}$ (processing method 1) for cases of varying airframe proximity. (Cases: $\Omega=5400$ RPM, BPF $=180$ Hz, $\left.W / c_{0.75 R}=\mathbf{1 . 0 8}\right)$

edge bluntness-vortex shedding (TEB-VS) self-noise. Therefore, it makes sense that the rod would not greatly affect this noise source mechanism because it physically occupies a very small fraction of the rotor disk area.

The tonal OASPL calculations for the experimental microphone measurements (processing method 2), denoted as $L_{T}$ and $L_{T, A}$, are provided in Figure 8 for the generic airframe cases of $W / c_{0.75 R}=1.08$ at all tested rotor-airframe proximities. As stated previously, these integrated tonal levels encompass the first $20 \mathrm{BPF}$ harmonics. The isolated rotor data are also provided for reference. Overall, the data show that the different airframe proximities have a noticeable effect on the data measured by microphones M3 through M5, which are at the lowest measured elevation angles below the rotor plane (see Table 1). Increasing the distance between the airframe and rotor tip from 0.1 to 0.2 tip radii below the rotor tip has the most drastic effect, with a maximum reduction of 


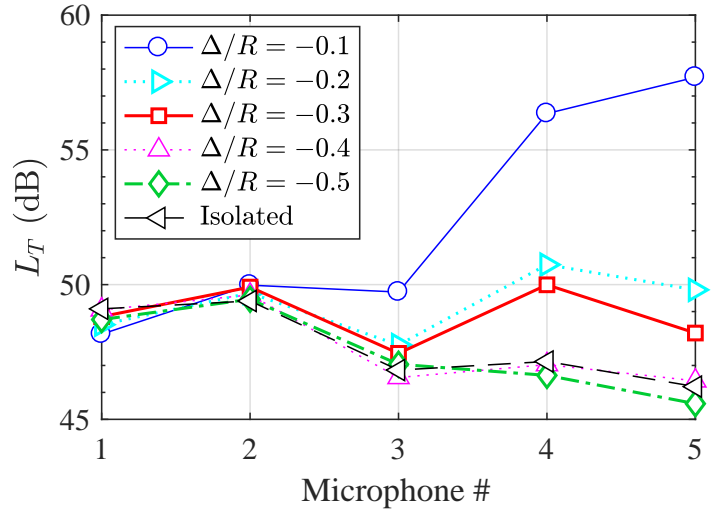

(a) Unweighted Tonal OASPL

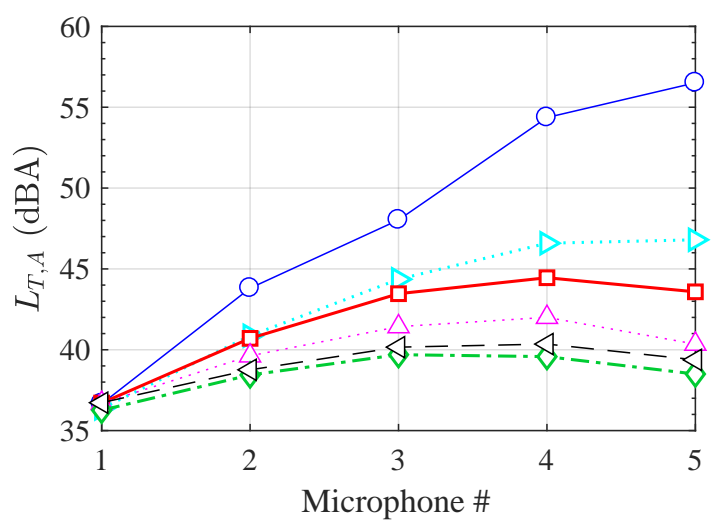

(b) A-weighted Tonal OASPL

Fig. 8. Tonal OASPLs of experimental measurements for different rotor tip clearances. (Cases: $\Omega=5400$ RPM, BPF $\left.=180 \mathrm{~Hz}, W / c_{0.75 R}=\mathbf{1 . 0 8}\right)$

approximately $8 \mathrm{~dB}$ in unweighted OASPL and nearly $10 \mathrm{~dB}$ in A-weighted OASPL at M5. Increasing this distance to 0.4 tip radii yields unweighted levels for all microphones that are nearly identical to those for the case of an isolated rotor. Increasing this further to 0.5 tip radii seems to decrease this further for microphones M4 and M5 below the levels for an isolated rotor; however, these levels are within the experimental uncertainty associated with the random FFT spectral representation of this data set. Despite the different levels resulting from the OAPSL weighting schemes, both Figures 8(a) and 8(b) show common overall trends of decreasing tonal integrated levels with increasing rotor-airframe vertical spacing. The results also show that the rotor-airframe case of $\Delta / R=-0.5$ is a condition in which integrated tonal noise levels are nearly identical to those of an isolated rotor using both OASPL weighting schemes.

Effects of Airframe Size The effects of varying airframe size on acoustic spectra and tonal OASPL are shown, respectively, in Figures 9 and 10. The acoustic spectra of Figure 9 show several prominent tones in the frequency range of 4.5 $\mathrm{kHz} \leq f \leq 6 \mathrm{kHz}$, which have a strong presence in the spectra for the motor only case. This is continuing proof that these tones are not related to the rotor-airframe aerodynamics.
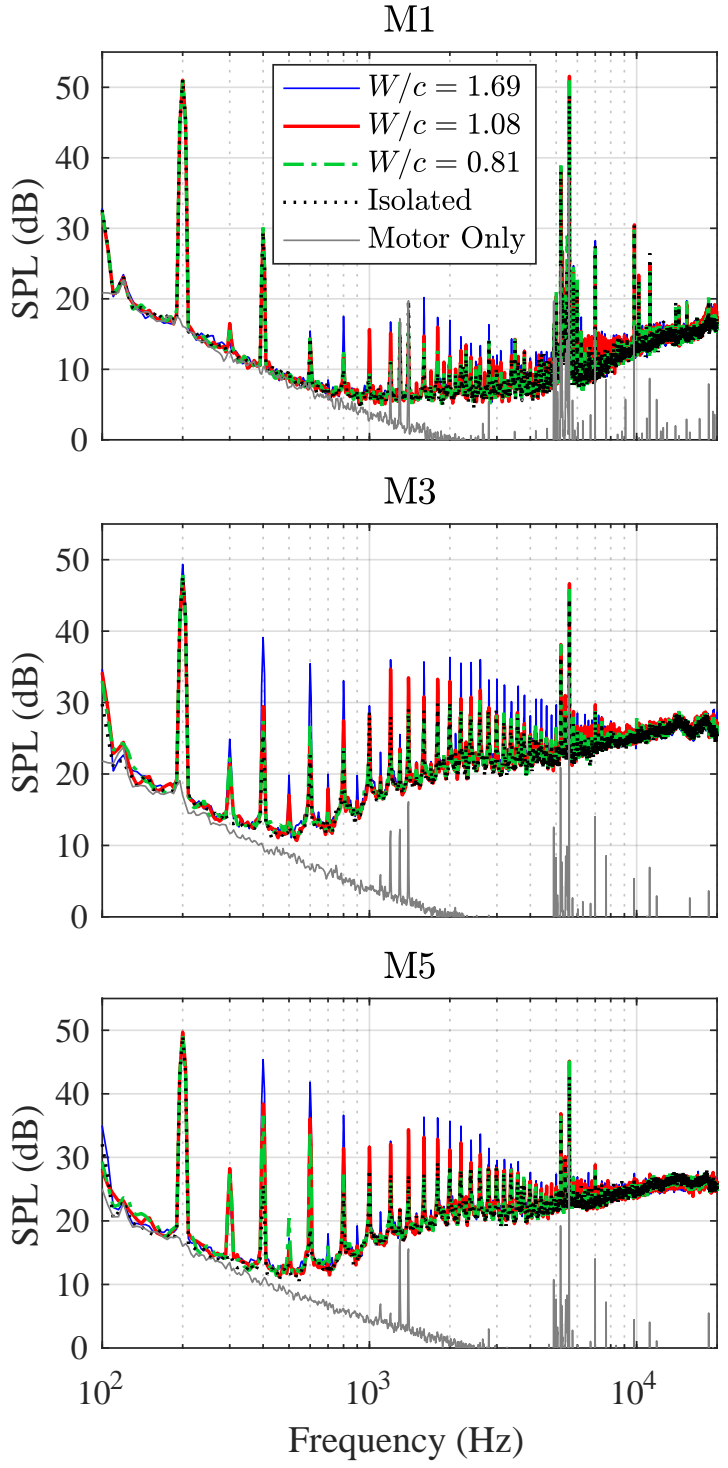

Fig. 9. Acoustic narrowband spectra at a frequency resolution of $5 \mathrm{~Hz}$ (processing method 1) for cases of varying airframe size. (Cases: $\Omega=6000 \mathrm{RPM}, \mathrm{BPF}=200 \mathrm{~Hz}, \Delta / R$ $=\mathbf{- 0 . 3 )}$

The M1 spectra for the different cases show very little difference in tonal character, which is also evident in the integrated tonal levels of Figure 10. The tonal OASPLs show a considerable noise reduction benefit in reducing the airframe size from $W / c_{0.75 R}=1.69$ to 1.08 , but much less of a benefit in reducing it further. It is interesting to note that the noise benefit is much more pronounced using the A-weighting metric in Figure 10(b). This is due to the fact that the largest tonal amplitude for these cases is the BPF itself, the A-weighted OASPL contribution of which is greatly reduced (see Figure 6). This amplifies the contributions of the higher BPF harmonics to the total integrated levels, especially because there is little difference in BPF amplitudes between the different cases considered.

Another interesting comparison to make is between the noise measurements associated with a generic, constant crosssection airframe and one with a varying cross-section. Figure 11 shows the tonal OASPL comparisons between the 


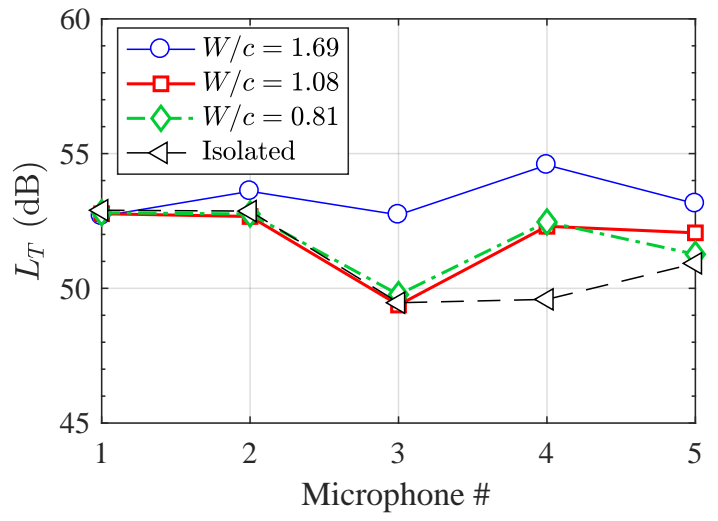

(a) Unweighted Tonal OASPL

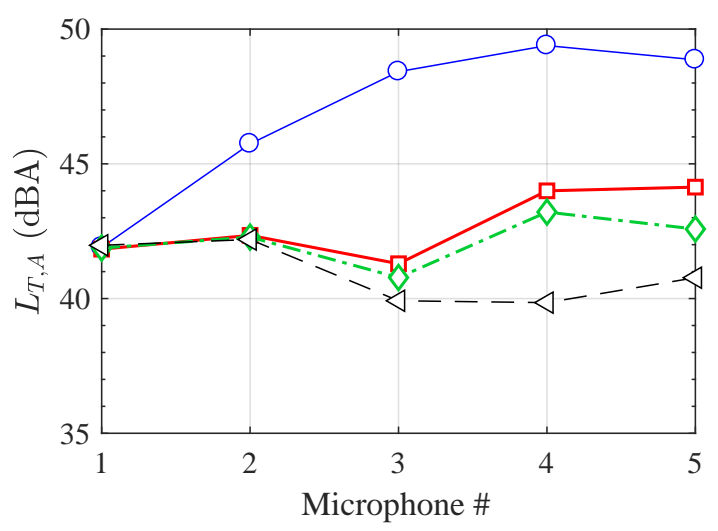

(b) A-weighted Tonal OASPL

Fig. 10. Tonal OASPLs of experimental measurements for different airframe sizes. (Cases: $\Omega=6000$ RPM, BPF $=$ $200 \mathrm{~Hz}, \Delta / R=\mathbf{- 0 . 3}$ )

generic airframe case of $W / c_{0.75 R}=1.08$ and the conical airframe for different rotor tip clearances. As these results show, the conical airframe cases typically exhibit higher unweighted overall levels for observers below the plane of the rotor (microphones M3-M5). One interesting exception to this trend occurs for the common case of $\Delta / R=-0.5$ (Figure 11(c)) in which both airframe configurations have nearly identical integrated levels at microphone M5. This observation is further contradicted by the A-weighted OASPL trend for the same cases in Figure 11(f), in which the conical airframe case has an OASPL approximately $6 \mathrm{~dB}$ greater than the generic rod airframe case.

To better make sense of these observations, acoustic narrowband spectra for microphone M5 are provided in Figure 12 for the different tip clearance cases. Starting with the first case of $\Delta / R=-0.1$, it can be seen that the conical airframe case exhibits higher tonal amplitudes than the rod airframe case for the first four BPF harmonics, then has a much sharper roll-off at higher frequencies. This helps explain why the unweighted OASPL at this location was higher for the conical airframe than the rod airframe, while the A-weighted OASPLs for the two cases are very similar. Moving on to the case of $\Delta / R=-0.3$, the two airframe cases are seen to display similar BPF harmonic content with the conical airframe case
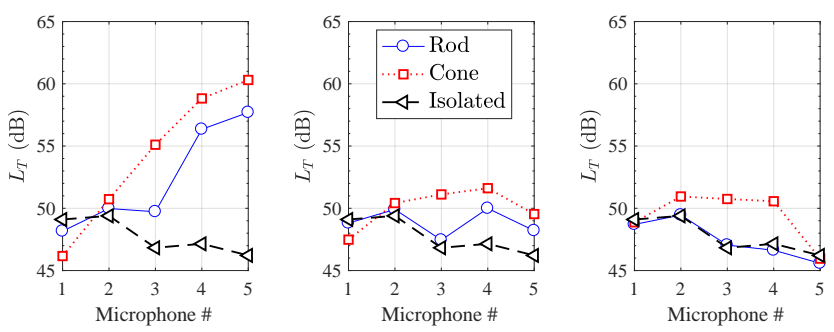

(a) $\Delta / R=-0.1$

(b) $\Delta / R=-0.3$

(c) $\Delta / R=-0.5$
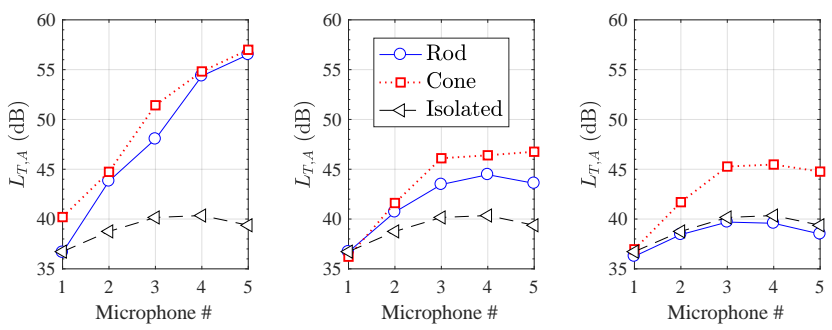

(d) $\Delta / R=-0.1$

(e) $\Delta / R=-0.3$

(f) $\Delta / R=-0.5$

Fig. 11. Tonal OASPL comparisons between $\operatorname{rod}(W / R=$ 1.08) and conical airframes for different tip clearances. Figures (a)-(c) represent unweighted OASPLs, (d)-(f) represent A-weighted OASPLs. (Cases: $\Omega=5400$ RPM) exhibiting consistently higher values. One interesting observation is that the BPF itself is seen to be drastically reduced for the conical airframe configuration as compared to both the rod airframe and isolated rotor cases. This is believed to be related to a near-field phase cancellation effect between the cone and airframe, which is investigated further in the next section. Finally, the case of $\Delta / R=-0.5$ reveals continued higher BPF harmonic content for the conical airframe case, with a considerable reduction (approximately $9 \mathrm{~dB}$ ) in the BPF acoustic amplitude. This behavior explains why the unweighted OASPL for this microphone location is comparable to the rod airframe and isolated rotor cases, while the A-weighted OASPL is considerably higher.

\section{CFD and Noise Predictions}

As stated previously, CFD-based noise predictions were carried out for a coarse set of rotor-airframe proximities for a fixed airframe cross-section of $W / c_{0.75 R}=1.08$ as well as a representative COTS configuration employing the conical airframe geometry (see Table 3). One important advantage of a FW-H prediction code is its ability to not only separate out the different noise terms of the acoustic analogy, but also to separate the noise contributions of different sources to the total radiated noise field. This is useful for the current cases in which both rotor and airframe surfaces warrant interrogation.

Mean Thrust Comparisons As an initial determination of the reliability of the OVERFLOW2 CFD data, mean thrust data were compared with the measured experimental data. A summary of the mean thrust data for the cases in common between experiments and predictions is provided in Table 4. It is important to note that experimental thrust data were only acquired on the rotor and not on the airframe geometries, while 

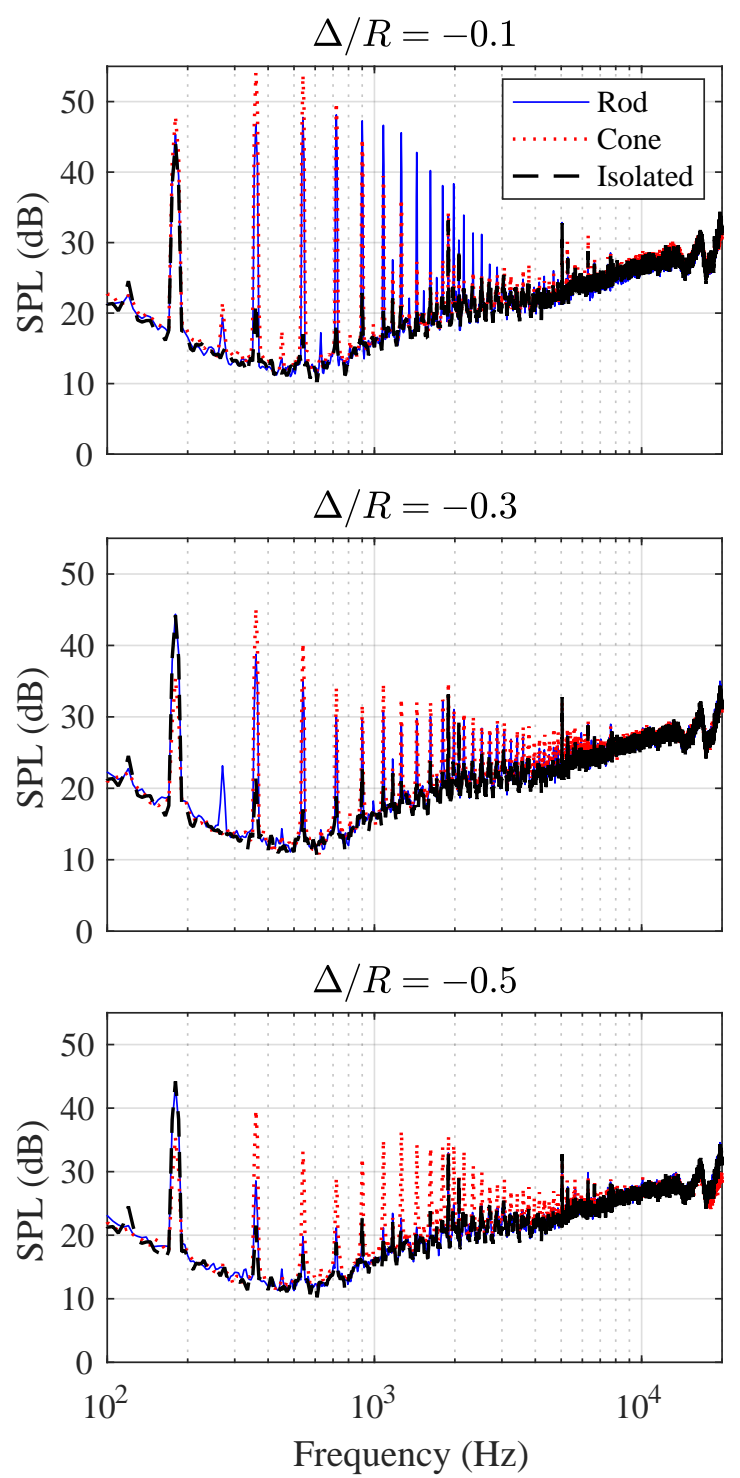

Fig. 12. Acoustic narrowband spectra (microphone M5) comparisons between $\operatorname{rod}\left(W / c_{0.75 R}=1.08\right)$ and conical airframe cases of varying rotor tip clearances. (Cases: $\Omega$ = 5400 RPM)

the CFD simulations acquired data for all surfaces. It is also worth noting that the uncertainties of the experimentally measured thrust data (provided in Table 4 for reference) were predominantly biased in nature due to the load cell itself. As the data show, there is good agreement between the measured and predicted thrust of the rotor across the different test cases. Specifically, all predicted rotor thrust levels are within a difference error relative to the experimental data of $4.1 \%$, the maximum difference occurring for the rotor-rod interaction case of $\Delta / R=-0.1$. As compared to the isolated rotor case, all rotor-airframe cases show a slight increase in rotor thrust levels as a result of a partial ground plane effect induced by the airframe. The most encouraging trend is that both measurements and predictions show the rod and cone cases of $\Delta / R=-0.1$ have the largest increase in rotor thrust levels. If attention is focused on the total predicted thrust generated by the different rotor-airframe combinations, the resulting drag effect caused by the airframes is evident. The largest reductions in thrust occur for the rotor-rod cases of $\Delta / R=-0.1$ and -0.3 , which is likely due to the large spanwise extent of the rod underneath the rotor plane. Some of this thrust loss is recovered for the rotor-cone case, which is most likely due to the increased spacing between rotor and airframe from tip to hub.

Table 4. Measured and predicted mean thrust levels.

\begin{tabular}{ll|r|rr}
\hline \hline & & \multicolumn{1}{|c|}{ Expt. $^{a}$} & \multicolumn{2}{|c}{ OVERFLOW2 } \\
Case & $\Delta / R$ & Rotor $(\mathrm{N})$ & Rotor $(\mathrm{N})$ & Total $(\mathrm{N})^{b}$ \\
\hline Isolated & N/A & 2.307 & 2.255 & 2.255 \\
Rod $^{c}$ & -0.1 & 2.365 & 2.269 & 2.105 \\
& -0.3 & 2.350 & 2.259 & 2.104 \\
& -0.5 & 2.339 & 2.259 & 2.133 \\
Cone & -0.1 & 2.369 & 2.290 & 2.153 \\
\hline \hline
\end{tabular}

${ }^{a}$ Experimental load cell bias uncertainty: $u_{b}= \pm 0.055 \mathrm{~N}$.

${ }^{b}$ Consists of rotor and airframe loading.

${ }^{c} W / c_{0.75 R}=1.08$.

High-Frequency Noise Content Prior to performing indepth comparisons between measured and predicted noise data, it is important to identify reasonable frequency limits for interrogation. This is because it is possible for CFD to predict artificially high-amplitude, higher frequency aerodynamic loading. To identify a suitable frequency range of comparison, acoustic spectra are compared between measurement and prediction for the rotor-rod interaction case of $W / c_{0.75 R}=$ 1.08 and $\Delta / R=-0.1$ at microphone M5, which is shown in Figure 13. As this figure shows, there is excellent agreement

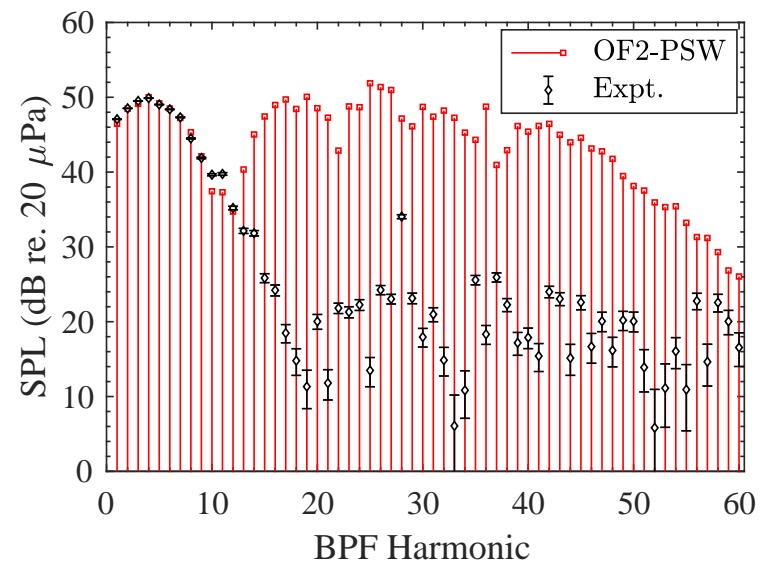

Fig. 13. BPF harmonic spectra comparison between OF2PSW and experimental data (microphone M5) for case of prominent rotor-rod interaction noise. (Case: $\Omega=\mathbf{5 4 0 0}$ RPM, $\Delta / R=\mathbf{- 0 . 1}$ )

between prediction and measurement for the first twelve BPF harmonics, after which OF2-PSW is seen to drastically overpredict the measured harmonic content. The causes of these over-predictions are currently unknown and will be investigated in future work. While the exact frequency at which this amplitude over-prediction will change depending on the simulation case, this case effectively elucidates the overall observed phenomenon. Therefore, the remaining acoustic comparisons between predictions and experimental measurements 
(in both time and frequency domains) will be done retaining the first ten BPF harmonics. Time domain representations of the acoustic predictions will be done using the filtering procedure mentioned previously.

Comparisons with Measurements Figure 14 presents rotor revolution time history and BPF harmonic spectra comparisons between OF2-PSW predictions and experimental measurements for the case of $\Delta / R=-0.1$. The results show very

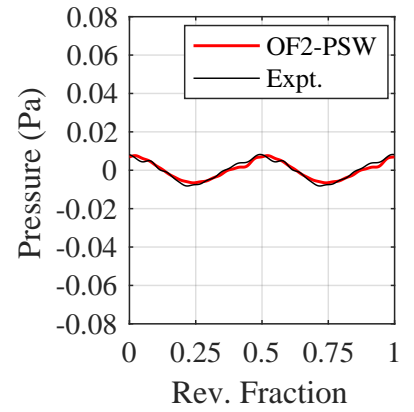

(a) M1 time history

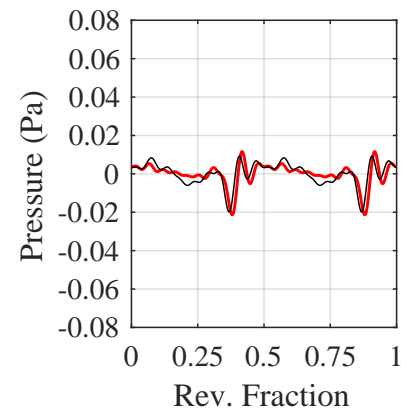

(c) M3 time history

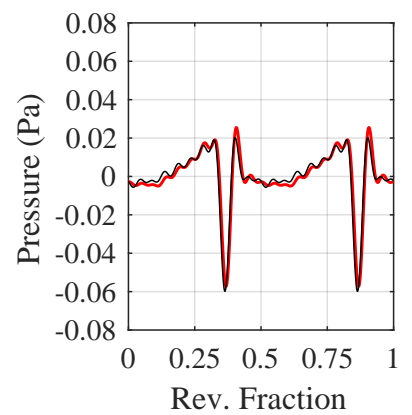

(e) M5 time history

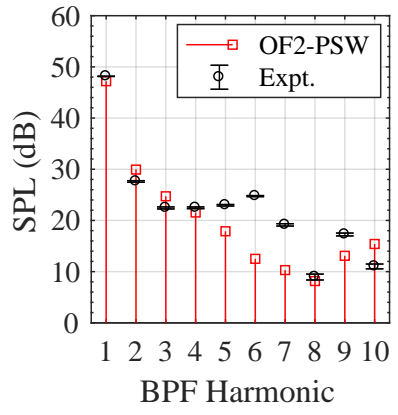

(b) M1 spectra

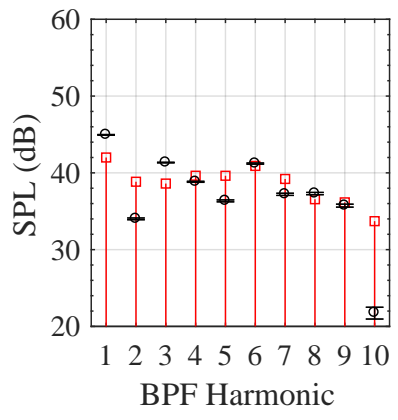

(d) M3 spectra

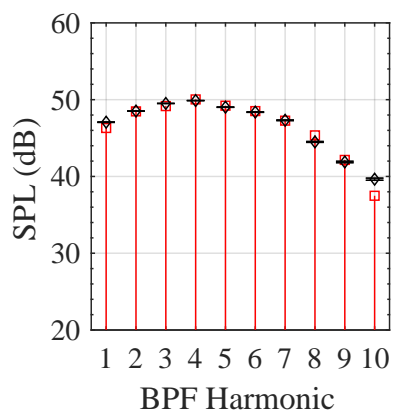

(f) M5 spectra
Fig. 14. Acoustic pressure time history and BPF harmonic spectra comparisons between OF2-PSW predictions and experimental measurements for generic rod airframe interaction case. (Cases: $\Omega=\mathbf{5 4 0 0} \mathbf{R P M}, \Delta / R=\mathbf{- 0 . 1}$ )

good overall agreement in both time and frequency domains. If attention is focused on the data for microphone M1 (Figures 14(a) and 14(b)), the rotor BPF is seen to be the dominant frequency component. The spectra show good agreement, except for the fifth through seventh BPF harmonics. The results for microphone M3 (Figures 14(c) and 14(d)) show an improvement in overall spectral amplitude comparisons between prediction and experiment, with the time domain prediction data properly displaying the negative and positive impulses observed in the experimental data. Finally, the data for microphone M5 (Figures 14(e) and 14(f)) show the best agreement, with all salient features of the experimental time history captured by the predictions and nearly identical spectral amplitudes shared between the two data sets.

Microphone M5 is selected for further comparisons between measurements and OF2-PSW predictions for the cases of varying airframe proximity to the rotor plane. Time history comparisons for the common tip clearance cases shared between the experimental and prediction cases are presented in Figure 15. As this figure shows, the mean revolution time

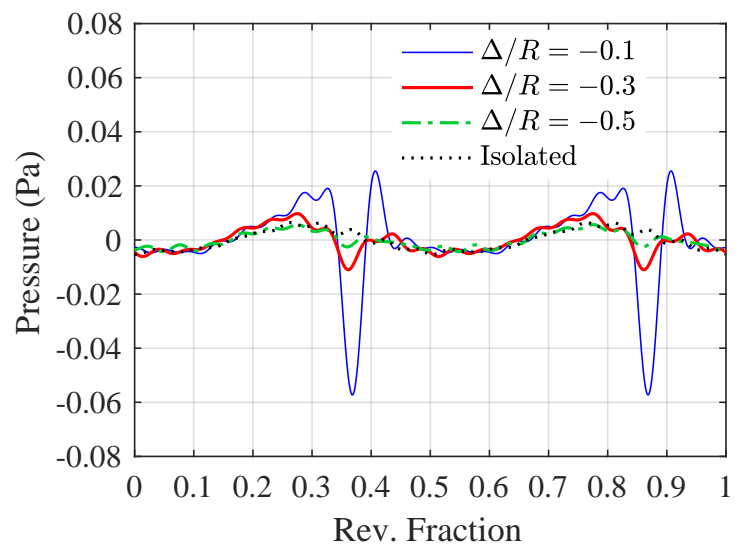

(a) M5 Prediction Time Histories

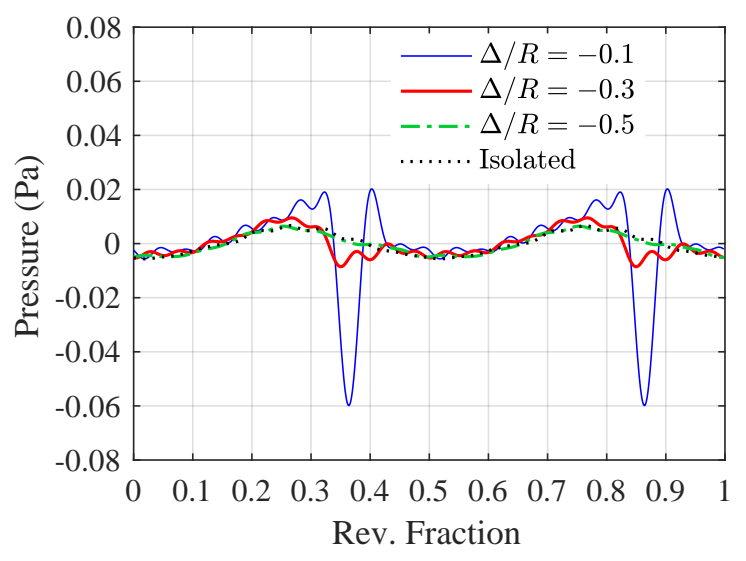

(b) M5 Measurement Time Histories

Fig. 15. Mean rotor revolution acoustic pressure time history comparisons between (a) OF2-PSW predictions and (b) experimental measurements for cases of varying airframe proximity to rotor plane. (Cases: $\Omega=5400$ RPM, $\left.W / c_{0.75 R}=\mathbf{1 . 0 8}\right)$

history trends compare reasonably well between predictions and measurements for all rotor tip clearance configurations. Furthermore, tonal OASPLs are also compared between predictions and measurements using the first ten BPF harmonics in Figure 16. The OASPL trends exhibited by the predictions and measurements are in reasonable agreement with one another. The largest discrepancies occur for the cases of the isolated rotor and for the rotor-rod case of $\Delta / R=-0.5$, where there is the least amount of acoustic content. However, the 
largest discrepancy in tonal OASPL between prediction and measurement, which occurs for microphone M5 and the case of $\Delta / R=-0.5$, is less than $2.2 \mathrm{~dB}$.

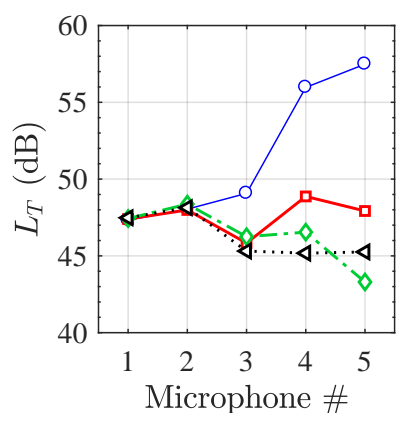

(a) $L_{T}$ Predictions

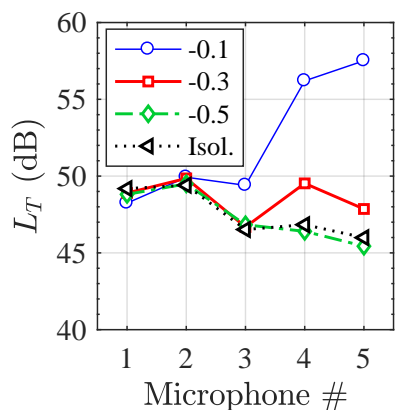

(b) $L_{T}$ Measurements
Fig. 16. Tonal OASPL comparisons between (a) OF2-PSW predictions and (b) experimental measurements for cases of varying airframe proximity to rotor plane. Note: First 10 BPF harmonics used in OASPL calculations. (Cases: $\Omega$ = 5400 RPM, $W / c_{0.75 R}=\mathbf{1 . 0 8}$ )

The conical airframe configuration also warrants consideration because it represents a more complex geometry. The time and frequency domain comparisons between predictions and measurements for microphones M1, M3, and M5 for the conical airframe case of $\Delta / R=-0.1$ are shown in Figure 17. As these results show, the agreement between prediction and experiment has degraded as compared to the previously discussed rod airframe case of Figure 14. While the overall time history trends are common between measurement and prediction, the OF2-PSW predictions contain a considerable amount of higher frequency harmonic content not contained within the experimental data. Despite this, however, observation of the BPF harmonic spectra of Figures 17(b), 17(d), and 17(f) show good agreement for the first four BPF harmonics. Despite the higher frequency discrepancies, the relatively high harmonic content at the in-plane observer (M1), as compared to the data for the previously discussed rod airframe case, is captured. In fact, the second largest harmonic amplitude of the M1 spectra for the rod airframe interaction case is 17 and $20 \mathrm{~dB}$ below the BPF harmonic amplitude for the prediction and measurement data, respectively. This is in sharp contrast to the current case, in which the 2 nd harmonic is within $4 \mathrm{~dB}$ of the BPF amplitude for both measurement and prediction data sets. It is further worth noting that the OF2-PSW time domain predictions are capturing the amplitudes of the pressure troughs for microphones M3 and M5 very well. Based on these results, it is believed that the OF2-PSW predictions are reliable for determining relative contributions of the rotor and airframe geometries to the radiated noise.

Rotor and Airframe Relative Noise Contributions As the previous section demonstrated, good agreement has been obtained between acoustic measurements and predictions for the various rotor-airframe interaction cases tested. In this section, the predictions are relied upon to identify the relative noise radiation roles of the rotor and airframes for the previously discussed cases.

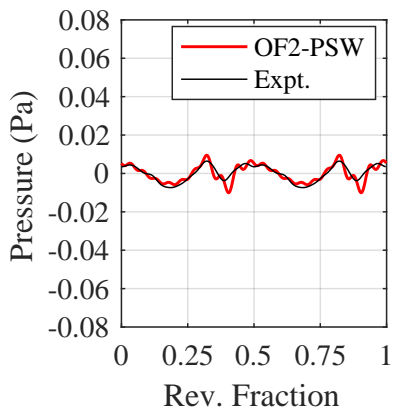

(a) M1 time history

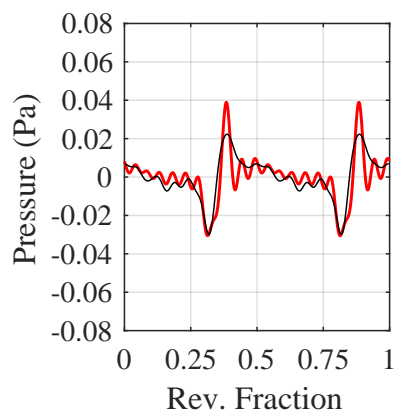

(c) M3 time history

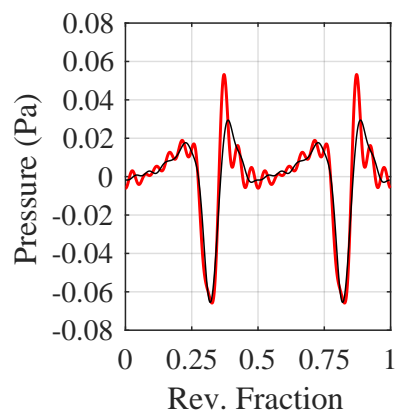

(e) M5 time history

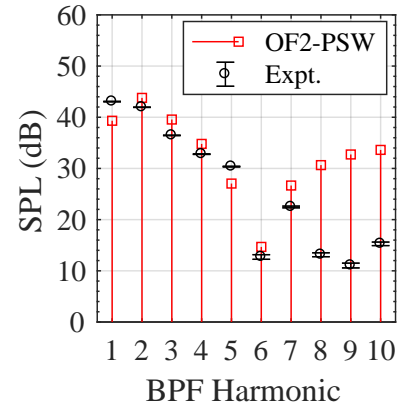

(b) M1 spectra

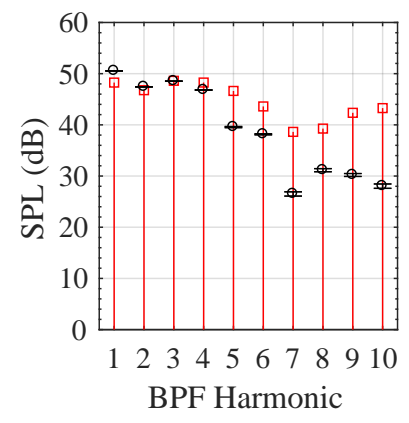

(d) M3 spectra

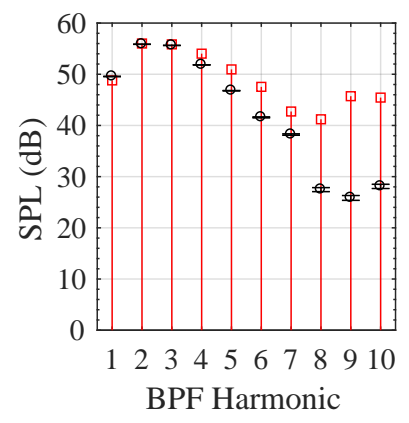

(f) M5 spectra
Fig. 17. Acoustic pressure time history and BPF harmonic spectra comparisons between OF2-PSW predictions and experimental measurements for conical airframe interaction case. (Cases: $\Omega=\mathbf{5 4 0 0}$ RPM, $\Delta / R=\mathbf{- 0 . 1}$ )

The computational surface contributions to time and frequency domain acoustic predictions for the rotor-rod interaction case of $\Delta / R=-0.1$ are shown in Figure 18 for microphones M1, M3, and M5. Starting with the in-plane observer, M1, it can be seen that the rotor blades are the dominant contributors. It makes physical sense that the rod does not contribute much to the noise at this location, because the M1 microphone is on the axis of the rod; therefore, there essentially are no surfaces that radiate noise in the direction of the microphone. Microphone M3, however, shows an interplay between the rotor blades and rod surfaces as they have a prominent phase cancellation effect for the 2 nd through 5 th BPF harmonics. This phase cancellation behavior continues for microphone M5; however, with a more prominent contribution of the rod surfaces to the radiated noise for the 3rd through 9th BPF harmonics. These results are indicative of the highly directive nature of the radiated sound for the cases 
of prominent rotor-airframe interaction noise.

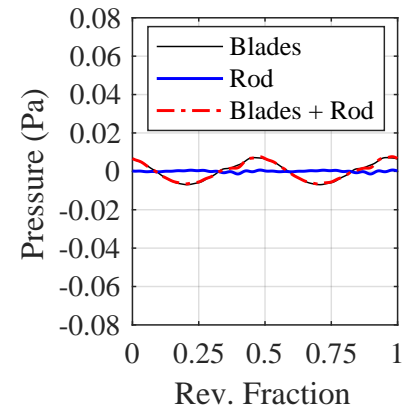

(a) M1 time history

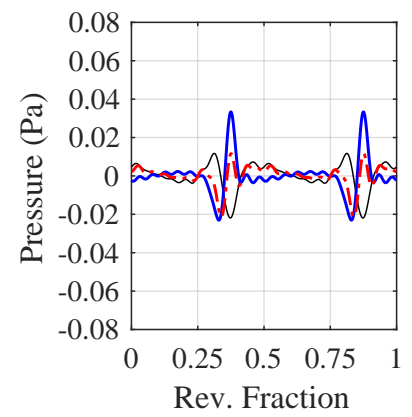

(c) M3 time history

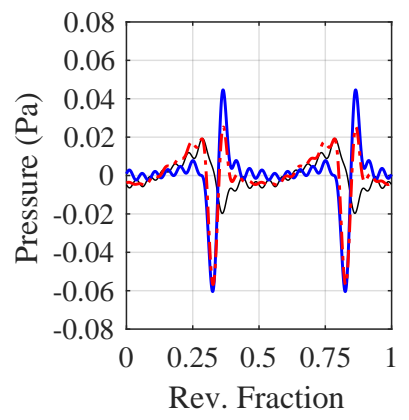

(e) M5 time history

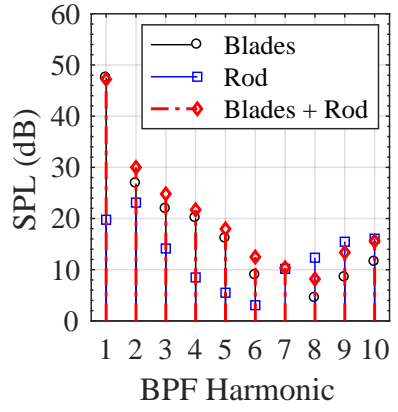

(b) M1 spectra

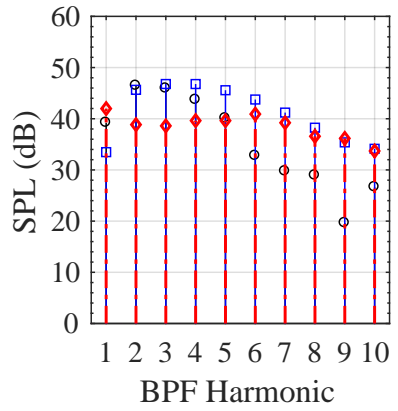

(d) M3 spectra

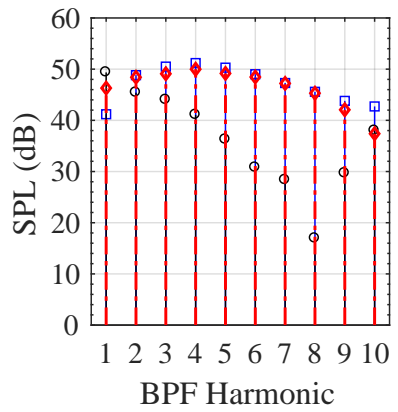

(f) M5 spectra
Fig. 18. Rotor blades and rod airframe surface contributions to predicted acoustic pressure time histories and BPF harmonic spectra. (Cases: $\Omega=5400$ RPM, $\Delta / R=\mathbf{- 0 . 1}$ )

Microphone M5 is further utilized to assess the roles of the different computational surfaces in rotor-airframe noise generation for different rotor tip clearances in Figure 19. As shown previously, the case of $\Delta / R=-0.1$ reveals a prominent negative pressure peak due almost solely to the rod surfaces, followed by a smaller amplitude positive pressure peak due to near-field phase cancellation effects between the rotor and rod surfaces. The case of $\Delta / R=-0.3$ reveals a drastic reduction in the amplitude of this negative peak, as well as in the amplitudes of the impulsive peaks due to the rotor blades. Increasing the spacing further to $\Delta / R=-0.5$ nearly eliminates the negative pressure peak of the rod surfaces, and further reduces the overall amplitudes of the rotor blade surfaces to more closely resemble the case of an isolated rotor (see Figure 15(a)).

For the sake of brevity, the conical airframe interaction

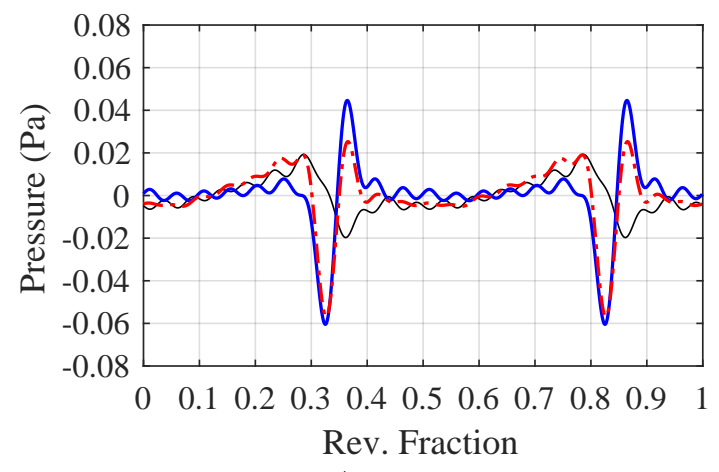

(a) $\Delta / R=-0.1$

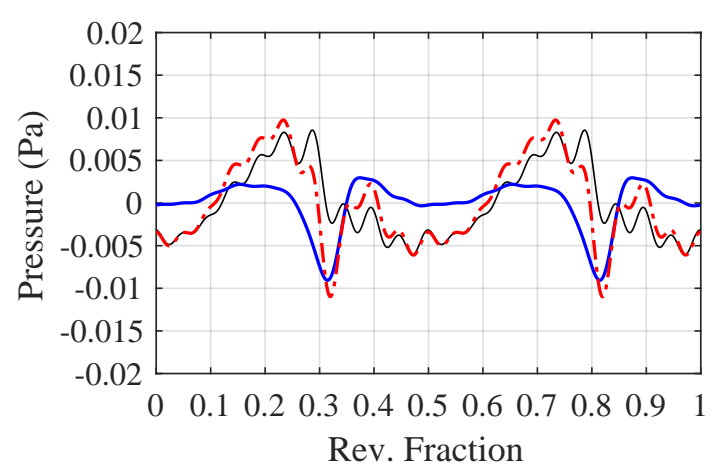

(b) $\Delta / R=-0.3$

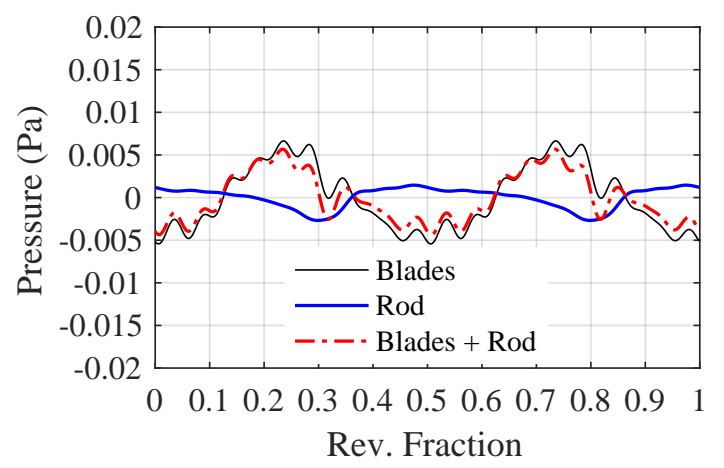

(c) $\Delta / R=-0.5$

Fig. 19. Contributions of rotor blades and rod airframe computational surfaces to time domain noise predictions (M5) for different tip clearance conditions. (Cases: $\Omega=$ 5400 RPM, $\left.W / c_{0.75 R}=\mathbf{1 . 0 8}\right)$

is presented only for microphone M1. This is because the trends observed for the other microphones are very similar to those observed for the previously discussed rotor-rod interaction case. As Figure 20 shows, the conical airframe is seen to play a much more important noise generation role as compared to the rod airframe. In fact, aside from the phase cancellation effect observed at the BPF, the cone surface is seen to be the predominant (and almost sole) contributor to the predicted noise for all other shown harmonics. These harmonic amplitudes are also considerably higher in amplitude as compared to the rod airframe case (see Figure 18(b)). This implies that the conical shape of the airframe makes it a more efficient noise radiator in the plane of the rotor.

Noise Source Mechanisms In this section, the physical sources of noise generation are investigated for the cases of 


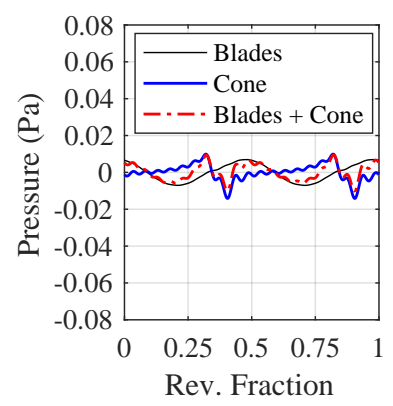

(a) M1 time history

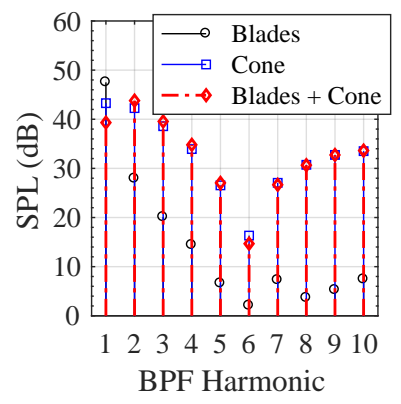

(b) M1 spectra

Fig. 20. Rotor blades and conical airframe surface contributions to predicted acoustic pressure time histories and BPF harmonic spectra. (Cases: $\Omega=5400 \mathrm{RPM}, \Delta / R=$ $-0.1)$

rotor-rod interactions. This is done by examining the noise source terms of the impermeable FW-H equation on the rotor and airframe surfaces as a function of observer time (Ref. 15). This type of analysis is believed to be very informative as to what mechanisms - such as pressure field interactions, vortex impingement - are responsible for rotor-airframe noise generation. As was discussed previously, a considerable amount of high-frequency, high-amplitude content is present in the predicted acoustics that is not representative of the experimental measurements. Furthermore, the FW-H code utilized in this study is not equipped with a causal time-domain filter for analyzing the surface noise source terms in a manner similar to that done in previous sections (such as for comparison with experiments). Therefore, the input CFD surface pressure data were downsampled by a factor of 4 . As a result of this downsampling, the higher harmonic content will roll off quickly as the Nyquist frequency is approached. However, because the purpose of this section is to qualitatively identify the noise source generation mechanisms responsible for the peak pressure events in the predicted time histories, this is considered to be acceptable.

To determine the viability of this downsampling technique, the revolution time history predictions for the harmonicallyfiltered and downsampled cases are compared for the rotorrod interaction cases of $\Delta / R= \pm 0.1$. Note that the case of $\Delta / R=+0.1$ is considered because it represents a case in which prominent interaction noise is expected and interaction mechanisms such as vortex impingement are not expected. Prior to examining the effects of downsampling the input surface pressure data, it is useful to examine the different predicted acoustic behaviors of these two configurations. Figure 21 presents comparisons of both the tonal OASPL predictions for the two rotor-rod interaction cases using the first 10 BPF harmonics for microphones M1-M5, as well as the BPF harmonic spectra for microphone M5. Note that the data shown in this figure are the total noise contributed by all surfaces in the CFD simulations. As this figure shows, the case of $\Delta / R=+0.1$ is predicted to generate considerably more noise than the case of $\Delta / R=-0.1$ for observer locations below the plane of the rotor. Explanations as to the reason for this drastic increase in noise levels for this configuration are provided in the following paragraphs in this section. Time history pre-

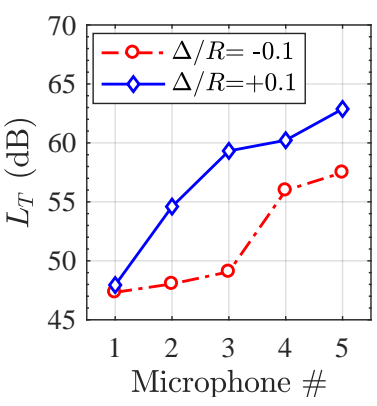

(a) $L_{T}$ predictions

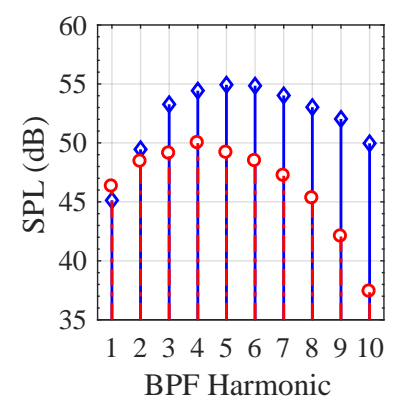

(b) M5 spectra
Fig. 21. Comparisons of (a) tonal OASPL and (b) M5 BPF harmonic spectra for rotor-rod interaction cases. Note: First 10 BPF harmonics used in OASPL calculations. (Cases: $\Omega=5400$ RPM, $\Delta / R= \pm 0.1$ )

dictions comparing the BPF harmonic filtering and downsampling techniques are shown in Figure 22 for both rod airframe interaction cases. Favorable agreement is obtained between

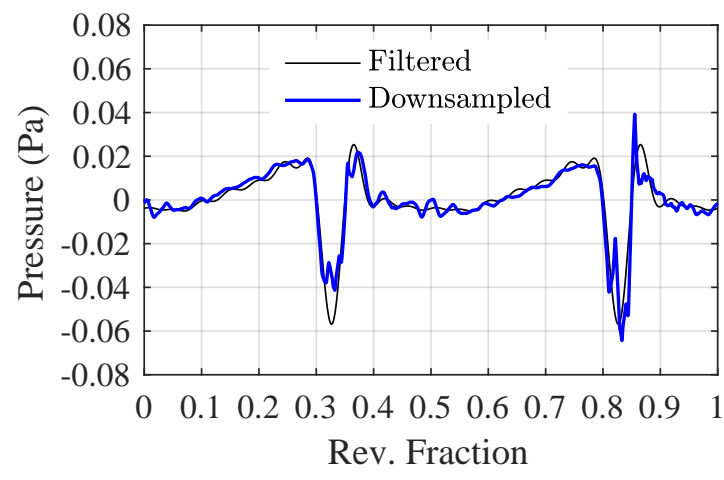

(a) $\Delta / R=-0.1$

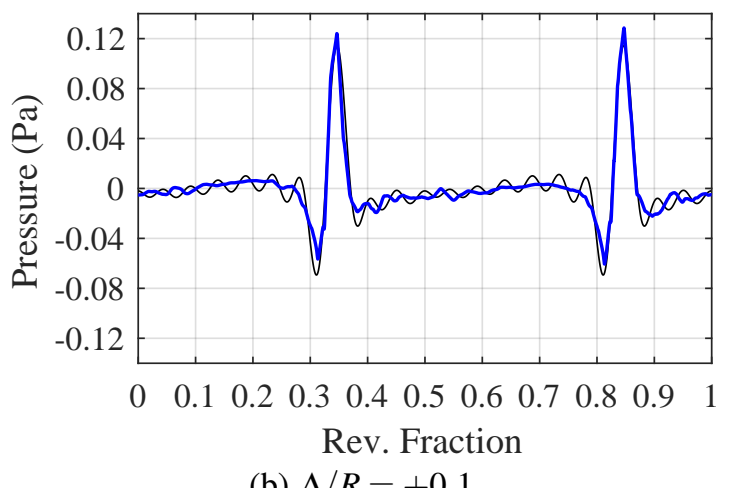

(b) $\Delta / R=+0.1$

Fig. 22. Microphone M5 prediction time history comparisons between BPF harmonic filtering and downsampling techniques. (Cases: $\Omega=5400$ RPM, $\left.W / c_{0.75 R}=\mathbf{1 . 0 8}\right)$

the two techniques, both of which show commonality in the peak-amplitude pressure events. Therefore, it is believed that the FW-H near-field surface pressure terms resulting from the downsampling technique can be relied upon to provide insight into the physical mechanisms responsible for these primary pressure events.

Contours of the total noise source terms on the rotor blades and rod airframe surfaces using an impermeable FW-H formulation are shown in Figure 23 at several discrete M5 observer 


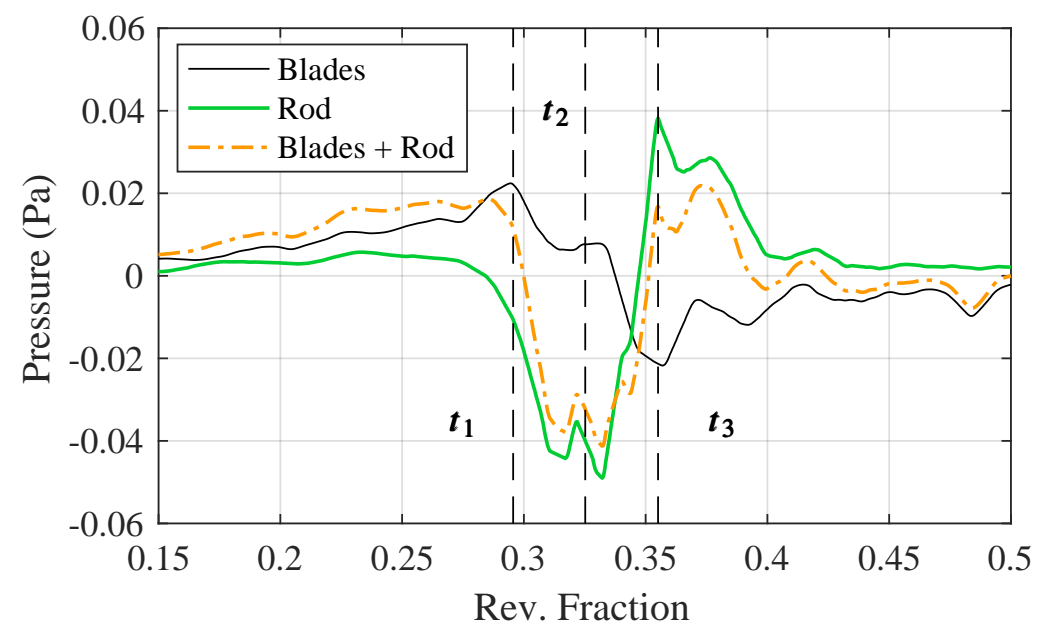

(a) M5 partial time history

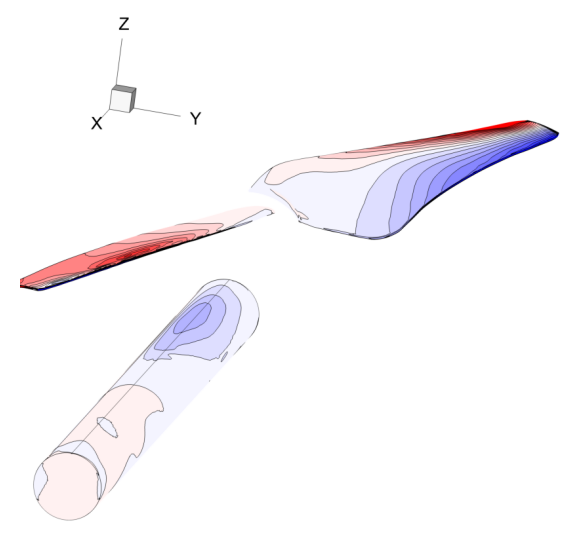

(b) Noise terms at $t_{1}$

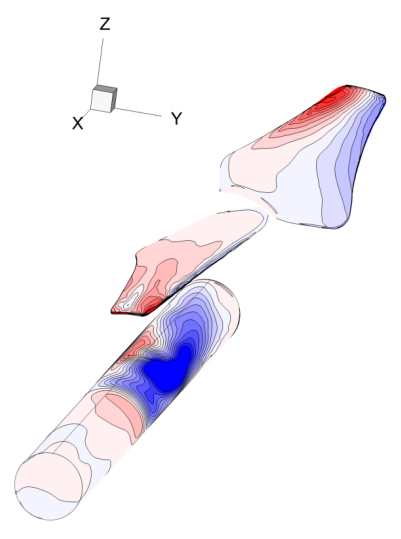

(c) Noise terms at $t_{2}$

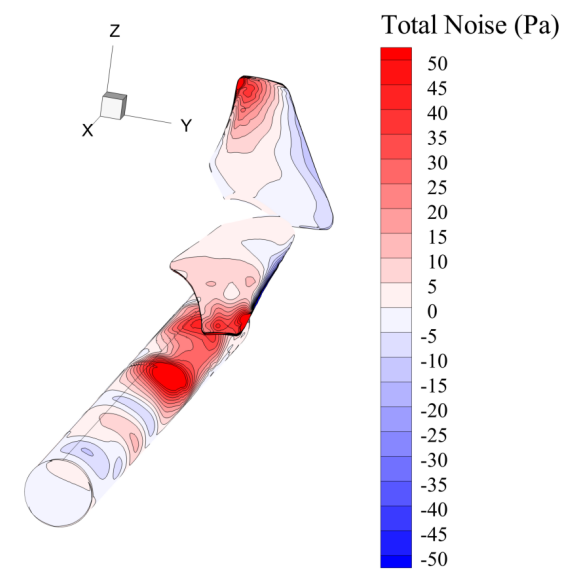

(d) Noise terms at $t_{3}$

Fig. 23. $\Sigma$ surfaces representing total noise impermeable FW-H source terms at different M5 observer times. (Cases: $\Omega$ $=5400 \mathrm{RPM}, \Delta / R=\mathbf{- 0 . 1})$

times. The contour plots of the noise sources are shown on surfaces of constant observer time, or " $\Sigma$ surfaces." That is, the sources at a given observer time are integrated to obtain the acoustic pressure time history at the given observer time. The time history showing one blade passage event being interrogated is provided in Figure 23(a). Note that the time histories shown in this figure are the result of the downsampling technique discussed previously and detail the individual contributions of the rotor blades and rod surfaces to the total radiated noise. At observer time $t_{1}$, the time history indicates that the rotor blades are producing a net positive acoustic pressure at microphone M5 due to pressure loading along the leading edge of the rotor blade as the blade approaches the rod surface. At observer time $t_{2}$, the same rotor blade is nearly parallel above the rod, which results in a large negative source loading on the rod surface. Due to the static nature of the rod, it can be assumed that this represents a time rate of change of noise due to the downwash induced by the potential field of the rotor blade. Finally, at observer time $t_{3}$, the rotor blade is beginning to clear the rod surface, and a large positive noise loading is observed on the rod surface, as well as a negative noise loading along the leading edge of the rotor blade. The large spanwise extent of the surface noise levels on the rod surface is believed to be evidence that this noise is not due to vortex impingement, but rather due to a time rate of change of the induced velocity generated by the potential field of the passing rotor blade.

The total noise predicted on the impermeable rotor and rod surfaces is further visualized in Figure 24 for the case of the rod positioned above the rotor, with the rod surface positioned at $\Delta / R=+0.1$. As the M5 observer time history of Figure 24(a) shows, the respective positive and negative pressure fluctuation events associated with the rotor blade and rod surfaces are almost perfectly out of phase with one another. While this does result in a slight reduction in the overall pressure time history, this configuration is still considerably louder than the case of $\Delta / R=-0.1$, which is evidenced by both the time history pressure amplitudes and shown contour levels in Figure 24. This is mostly due to the positive pressure pulse associated with the rod at $t_{2}$. The images shown in Figures 24(b) 


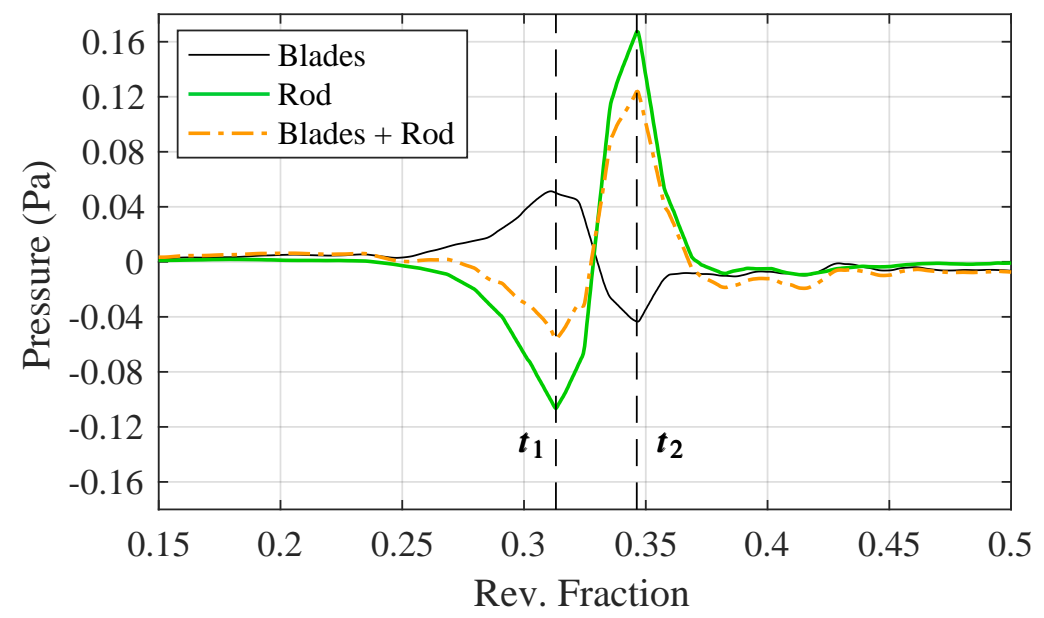

(a) M5 partial time history

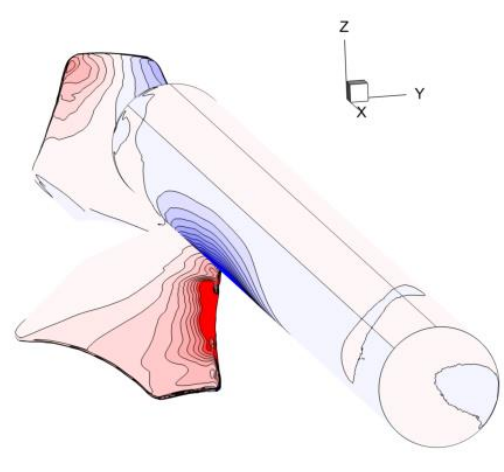

(b) Noise terms at $t_{1}$ (upper view)

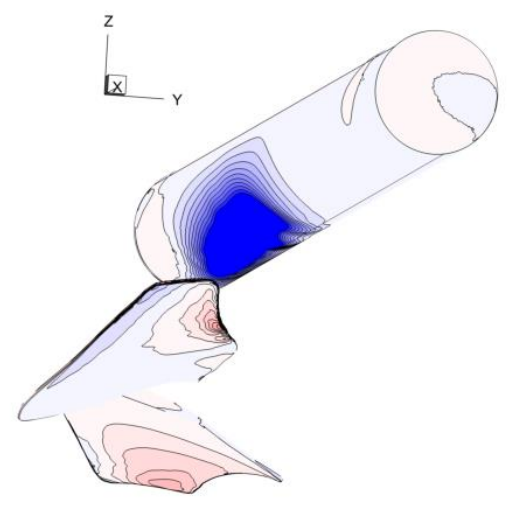

(c) Noise terms at $t_{1}$ (lower view)

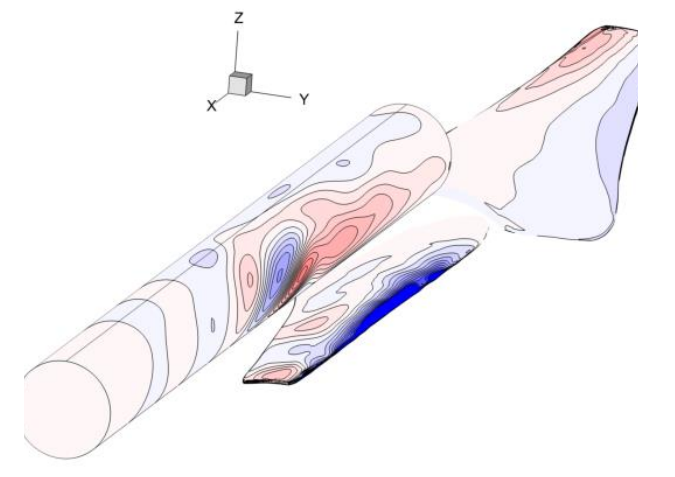

(d) Noise terms at $t_{2}$ (upper view)

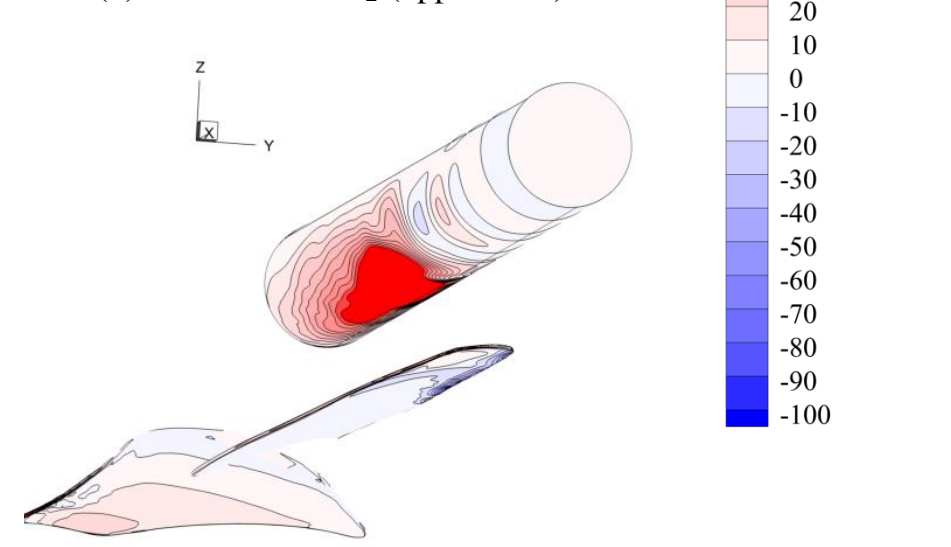

(e) Noise terms at $t_{2}$ (lower view)

Total Noise $(\mathrm{Pa})$

Fig. 24. $\Sigma$ surfaces representing total noise impermeable FW-H source terms at different M5 observer times. (Cases: $\Omega$ $=5400 \mathrm{RPM}, \Delta / R=+\mathbf{+ 0 . 1})$

and 24(c) represent the total noise source contributions of the rotor blade and rod surfaces from different vantage points at time $t_{1}$. Similar to the previous case of $\Delta / R=-0.1$, this time instance shows positive acoustic pressure generated along the leading edge of the rotor blade and prominent negative acoustic pressure along the lower surface of the rod. At time instant $t_{2}$, the rotor blade is nearly parallel with the rod, and the noise amplitudes of the rotor blade and rod surfaces have switched signs. While the rotor blade exhibits noise amplitudes at this time instance that are comparable to those at the previous time instance, the rod-generated noise is higher in amplitude.

Regardless of whether the rod is located above or below the rotor plane, the unsteady loading noise that occurs on the rotor blades is due to a change in induced velocity generated 
by each rotor blade as it encounters the pressure potential field of the rod. As the rotor blade passes the rod, there is an upwash and downwash effect caused by the bound vortex of the rotor blades, the resultant pressure field of which causes load fluctuations on the rod. The fact that considerably more noise is generated when the rod is above the plane of the rotor is attributed to higher rates of change in induced velocity on the upper surface of the rotor blade. This is analogous to the pressure field around a lifting airfoil, in which the upper surface (suction side) typically encounters considerably higher negative pressure amplitudes than the positive amplitudes on the lower surface (pressure side). This explanation of the noise mechanism is further supported by the notion that the loads induced on the rod due to downwash caused by the rotor blades would have the same direction, regardless of which side of the rotor plane the rod is located. This is evidenced by the fact that the signs of the rod-generated acoustic pulses are the same for both cases.

\section{CONCLUSIONS AND FUTURE WORK}

This study displays experimental measurements and CFDbased acoustic predictions of several simplified rotor-airframe configurations representative of rotary-wing UAS in ideal hover conditions. Experimental measurements revealed prominent tonal noise associated with airframes within close proximity of the rotor plane, which was observed to decay fairly rapidly with increasing rotor-airframe spacings. Cases of prominent rotor-airframe interaction noise were found to be highly directive as a function of observer azimuth, the highest amplitudes of which were exhibited by observers located out of the centerplane of the airframe. Generic airframes of constant cross-section were found to have little or no effect on noise radiated in the plane of the rotor, while a conical airframe of varying cross-section revealed increased harmonic acoustic content at the same observer location. These observations served as initial indications that the airframes themselves can serve as important and prominent noise contributors under certain conditions. Analysis of the different rotorairframe conditions using unweighted and A-weighted tonal OASPL metrics revealed similar overall trends, but of very different amplitude depending on the frequency ranges over which the tonal content was present. In addition, the broadband noise content was observed to be nearly identical for all tested rotor-airframe configurations, providing evidence that this noise is due to rotor-generated turbulence because the airframes tested occupy a very small azimuthal portion of the rotor disk area.

Tonal acoustic predictions incorporating CFD-calculated rotor and airframe surface pressures into an impermeable FW$\mathrm{H}$ solver were found to compare favorably with experimental measurements. Filtering of the predicted acoustic data was required, however, for time domain comparisons with the experimental data due to artificially high-frequency, high-amplitude predicted acoustic content. These favorable comparisons motivated investigation of the relative noise-generating roles of the rotor blade and airframe surfaces. The case of a generic rod airframe in close proximity to the rotor plane revealed that the airframe plays a very important role in generating BPF harmonic noise out of the plane of the rotor. This exact role is highly directive; however, this depends on the relative phasing with the rotor itself. The conical airframe case examined presented a similar out-of-plane trend; however, it also showed that the airframe generates the majority of higher BPF harmonic noise in the plane of the rotor. This is attributed to the conical airframe being a more efficient noise radiator in the plane of the rotor, because the airframe surface is better aligned with the in-plane observer. Downsampling of the CFD surface pressure data input into the FW-H solver allowed for a qualitative analysis of the on-surface noise terms for both rotor and rod surfaces as a function of observer time. This technique revealed overall similar noise prediction behavior between the cases of a generic rod airframe being located above and below the plane of the rotor; however, the case of the rod above the rotor plane is predicted to generate considerably more noise.

Future experimental testing is planned using airframe shapes of greater complexity, including wing sections to represent tiltrotor and tilt-wing configurations. Acoustic wind tunnel testing on representative multi-copter platforms is also planned to identify the effects of forward flight on rotorairframe interaction noise. Aeroacoustic predictions will also be conducted to emulate these configurations. Investigations of the sources of the artificially high-amplitude, highfrequency content present in the acoustic predictions will also be conducted.

\section{Author contact}

Nikolas S. Zawodny, nikolas.s.zawodny@ nasa.gov

\section{ACKNOWLEDGMENTS}

The authors would like to acknowledge Mr. Matthew Hayes of the Structural Acoustics Branch at NASA Langley Research Center for his assistance with experimental setup and testing. The authors would also like to acknowledge Dr. Eric Greenwood of the NASA Langley Aeroacoustics Branch for his assistance with developing deterministic noise analysis techniques. This work was funded by the NASA Convergent Aeronautical Systems (CAS) sub-project, the Design Environment for Novel Vertical Lift Vehicles (DELIVER), the principal investigator of which is Dr. Colin Theodore.

\section{REFERENCES}

${ }^{1}$ FAA, Fact Sheet - Unmanned Aircraft Systems (UAS), Available at: http://www.faa.gov/news/fact_sheets/news_story .cfm?newsId=18297, 2015.

${ }^{2} 112$ th Congress, Subtitle B - Unmanned Aircraft Systems, Public Law 112-95, 2012.

${ }^{3}$ Kesselman, S., The First 1,000 Commercial UAS Exemptions, Association for Unmanned Vehicle Systems International (AUVSI), 2016. 
${ }^{4}$ Zawodny, N. S., Boyd Jr., D. D., and Burley, C. L., "Acoustic Characterization and Prediction of Representative , SmallScale Rotary-Wing Unmanned Aircraft System Components," AHS International 72nd Annual Forum, 2016.

${ }^{5}$ Farassat, F., Derivation of Formulations 1 and $1 \mathrm{~A}$ of Farassat, Hampton, VA, NASA TM 2007-214853, 2007.

${ }^{6}$ Boyd, D. D., "HART-II Acoustic Predictions using a Coupled CFD / CSD Method," American Helicopter Society 65th Annual Forum, 2009.

${ }^{7}$ Nguyen, L. C. and Kelly, J. J., A Users Guide for the NASA ANOPP Propeller Analysis System, Hampton, VA, NASA CR 4768, 1997.

${ }^{8}$ Burley, C. L. and Brooks, T. F., "Rotor Broadband Noise Prediction with Comparison to Model Data," Journal of the American Helicopter Society, Vol. 49, (1), 2004, pp. 28-42. doi: 10.4050/JAHS.49.28

${ }^{9}$ Coffen, C. D., Tilt Rotor Hover Aeroacoustics, Ithaca, NY, NASA CR 177598, 1992.

${ }^{10}$ Rutledge, C. K., Coffen, C. D., and George, A. R., A Comparative Analysis of XV-15 Tiltrotor Hover Test Data and WOPWOP Predictions Incorporating the Fountain Effect, Hampton, VA, NASA CR 189455, 1991.

${ }^{11}$ Grosveld, F. W., "Calibration of the Structural Acoustics Loads and Transmission Facility at NASA Langley Research Center," InterNoise 99, 1999.

${ }^{12}$ Chan, W. M., Gomez III, R. J., Rogers, S. E., and Buning, P. G., "Best Practices in Overset Grid Generation," 32nd AIAA Fluid Dynamics Conference, 2002.

${ }^{13}$ Bendat, J. S. and Piersol, A. G., Random Data Analysis and Measurement Procedures, John Wiley \& Sons Inc., third edition, 2000, Chapter 6: Statistical Errors in Advanced Estimates, pp. 316-348.

${ }^{14}$ Bennett, R. L. and Pearsons, K. S., Handbook of Aircraft Noise Metrics, Canoga Park, CA, NASA CR 3406, 1981.

${ }^{15}$ Farassat, F., Theory of Noise Generation from Moving Bodies with an Application to Helicopter Rotors, Hampton, VA, NASA TR R-451, 1975. 